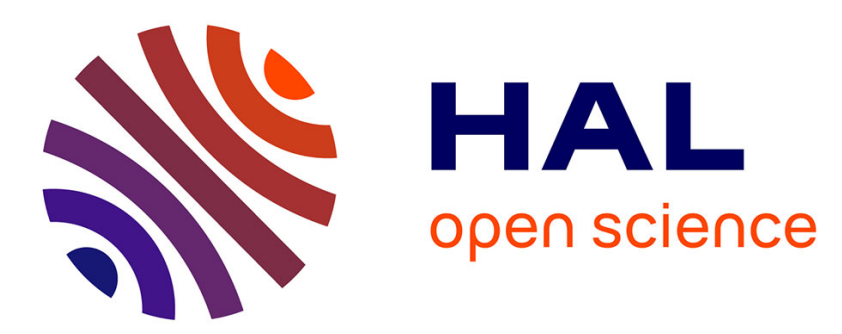

\title{
A stabilized Lagrange multiplier finite-element method for flow in porous media with fractures
}

Markus Köppel, Vincent Martin, Jean E. Roberts

\section{To cite this version:}

Markus Köppel, Vincent Martin, Jean E. Roberts. A stabilized Lagrange multiplier finite-element method for flow in porous media with fractures. GEM - International Journal on Geomathematics, In press, 10.1007/s13137-019-0117-7 . hal-01761591v3

\section{HAL Id: hal-01761591 \\ https://hal.science/hal-01761591v3}

Submitted on 29 Dec 2018

HAL is a multi-disciplinary open access archive for the deposit and dissemination of scientific research documents, whether they are published or not. The documents may come from teaching and research institutions in France or abroad, or from public or private research centers.
L'archive ouverte pluridisciplinaire HAL, est destinée au dépôt et à la diffusion de documents scientifiques de niveau recherche, publiés ou non, émanant des établissements d'enseignement et de recherche français ou étrangers, des laboratoires publics ou privés. 


\author{
Markus Köppel • Vincent Martin •
}

Jean E. Roberts

\title{
A stabilized Lagrange multiplier finite-element method for flow in porous media with fractures
}

April 2018

\author{
M. Köppel \\ Universtität Stuttgart, Institut für Angewandte Analysis und Numerische \\ Simulation (IANS), Pfaffenwaldring 57, 70569 Stuttgart, Germany, E-mail: \\ markus.koeppel@ians.uni-stuttgart.de \\ V. Martin \\ Université de Technologie de Compiègne (UTC), Laboratoire de Mathématiques \\ Appliquées de Compiègne (LMAC), Rue du docteur Schweitzer CS 60319, 60203 \\ Compiègne Cedex France, E-mail: vincent.martin@utc.fr \\ J. E. Roberts \\ Inria Paris, 2 rue Simone Iff, 75589 Paris \& Université Paris-Est, CERMICS (ENPC), \\ 77455 Marne-la-Vallée 2, France, E-mail: jean-elizabeth.roberts@inria.fr
}

\begin{abstract}
In this work we introduce a stabilized, numerical method for a multidimensional, discrete-fracture model (DFM) for single-phase Darcy flow in fractured porous media. In the model, introduced in an earlier work, flow in the $(n-1)$-dimensional fracture domain is coupled with that in the $n$-dimensional bulk or matrix domain by the use of Lagrange multipliers. Thus the model permits a finite element discretization in which the meshes in the fracture and matrix domains are independent so that irregular meshing and in particular the generation of small elements can be avoided. In this paper we introduce in the numerical formulation, which is a saddle-point problem based on a primal, variational formulation for flow in the matrix domain and in the fracture system, a weakly consistent stabilizing term which penalizes discontinuities in the Lagrange multipliers. For this penalized scheme we show stability and prove convergence. With numerical experiments we analyze the performance of the method for various choices of the penalization parameter and compare with other numerical DFM's.
\end{abstract}

Keywords discrete fracture model · finite element method · stabilized Lagrange multiplier method $\cdot$ penalization $\cdot$ nonconforming grids 


\section{Introduction}

Fractures represent one of the most challenging heterogeneities for the approximation of fluid flow in porous media. Typically their lateral dimension is considerably smaller compared to their extensions in other directions. Moreover fractures may act as barriers to and/or conduits for fluid flow. Depending on the hydrogeological properties and the scale of consideration, the presence of fractures thus may lead to a significant change in the flow behavior in the subsurface. Because fault zones occur in many applications, such as $\mathrm{CO}_{2}$ sequestration, underground storage of radioactive waste and enhanced oil recovery, the consideration of fractures in modeling of flow in porous media has received more and more attention in the last decades. A variety of different models have been proposed.

A common way to incorporate fractures in models is the discrete-fracture (DFM) approach, in which information concerning the fracture location in the domain of interest is required, and the fluid flow in the fracture as well as in the surrounding domain is calculated. In this context the fractures are often considered as $(n-1)$-dimensional objects within the surrounding $n$-dimensional matrix domain in order to avoid the generation of small elements of the spatial discretization grid. Such models have been studied, in e.g. (Alboin et al, 2002; Angot et al, 2009), assuming Darcy flow in both, fracture and matrix, parts of the domain. Other studies addressed Forchheimer flow in the fractures (Knabner and Roberts, 2014) or Darcy-Brinkman flow (Lesinigo et al, 2011). Multiphase flow has also been considered, e.g. (Ahmed et al, 2017; Brenner et al, 2015; Hoteit and Firoozabadi, 2008). Some articles deal with discrete fracture network (DFN) models, e.g. (Berrone et al, 2014; Pichot et al, 2012). Whereas some of these models are based on finite element methods, (Baca et al, 1984), others use mixed or mixed-hybrid finite elements, (Boon et al, 2018; Martin et al, 2005), finite volume methods, (Fumagalli et al, 2016; Karimi-Fard et al, 2004; Reichenberger et al, 2006), multi-point flux methods, (Sandve et al, 2012), or mimetic finite difference methods, (Antonietti et al, 2016b; Formaggia et al, 2018), or discontinuous Galerkin methods, (Antonietti et al, 2016a; Massing, 2017), to discretize the problem.

For discretization schemes, in what may be referred to as a matching fracture and matrix grid approach, the fracture mesh elements coincide with faces of the matrix mesh elements. However one may wish to discretize the fracture more finely in the case of a highly conductive fracture or more coarsely in the case of a barrier. Therefore it may be necessary to use methods allowing for non-matching grids; see e.g. (Chave et al, 2018; Faille et al, 2016; Frih et al, 2012). Still with these methods the matrix grid must be aligned with the fracture. By contrast, with nonconforming methods a fracture can cut through the interior of matrix elements because of an independent meshing of the corresonding domains. This can be achieved, for example, with locally enriched basis functions in the vicinity of the fracture to account for the resulting discontinuities, in what is commonly referred to as an extended finite element method (XFEM), e.g. in (Fumagalli and Scotti, 2013; Schwenck et al, 2015).

This work presents an alternative nonconforming discretization scheme for a model, introduced in Köppel et al (2018), for single-phase, Darcy flow in frac- 
tured porous media. The model uses Lagrange multiplier variables, which represent a local fluid exchange between fracture and matrix, in a primal variational formulation. The new numerical scheme, like that of Köppel et al (2018), uses continuous piecewise linear or bilinear approximations for the pressure both in the matrix and in the fracture and piecewise constant functions to approximate the multipliers. Here however, following ideas of Burman and Hansbo (2010a), we add a stabilization term which penalizes jumps in the multipliers over regular portions of the fracture. The permeability in the fracture is assumed to be larger than that in the matrix. Hence the fluid pressure is continuous excluding the case of a geological barrier, which will be subject of future research. Because of the use of the multiplier this model allows for mutually independent grids of the matrix and the fracture, both discretized with continuous, piecewise-(bi)linear basis functions. As in Köppel et al (2018), the Lagrange multiplier is discretized by means of discontinuous, piecewise-constant, basis functions, though here the multipliers are no longer associated with an independent but size-constrained grid but with a grid generated by intersections of the matrix grid with the fracture. Following Burman and Hansbo (2010a), we add a weakly consistent stabilizing term which penalizes the jumps of the discrete multipliers. This leads to a stabilization of the discrete saddle point system and thus reduces the condition numbers involved. In Section 2, we recall briefly the continuous formulation of the Lagrange multiplier method. Section 3 concerns the discrete formulation of the problem. We introduce a weakly consistent penalty term to stabilize the discrete system, prove the stability of the discrete formulation and its convergence under conditions on the regularity of the Lagrange multiplier. In Section 4, the theoretical findings are analyzed numerically by means of several numerical experiments, including two benchmarks from Flemisch et al (2018), validating the method. Finally we conclude and discuss the proposed method in Section 5.

\section{The continuous formulation for the Lagrange multiplier model}

In this section we recall briefly the continuous model for the Lagrange-multiplier DFM, introduced in Köppel et al (2018). Let $\Omega$ be a domain in $\mathbb{R}^{2}$, representing a porous medium and let $\gamma \subset \Omega$ be an one-dimensional surface representing a fracture. The extension to $3 \mathrm{D}$ does not pose real conceptual difficulties for the analysis though. Let $\mathbf{n}_{\gamma}$ denote one of the two possible continuous unit vector fields on $\gamma$, and let $\mathbf{K}$ and $\mathbf{K}_{\gamma}$ be symmetric, uniformly positive-definite, bounded, permeability tensor fields on $\Omega$ and $\gamma$ respectively, with constants $C_{\mathbf{K}}^{M}$ and $C_{\mathbf{K}}^{m}>0$ such that

$$
\begin{array}{ll}
\|\mathbf{K}(x)\| \leq C_{\mathbf{K}}^{M}, \quad \forall x \in \Omega, & C_{\mathbf{K}}^{m}\|\mathbf{v}\|_{0, \Omega} \leq(\mathbf{K} \mathbf{v}, \mathbf{v})_{\Omega}, \quad \forall \mathbf{v} \in L^{2}(\Omega), \\
\left\|\mathbf{K}_{\gamma}\left(x_{\gamma}\right)\right\| \leq C_{\mathbf{K}}^{M}, \quad \forall x_{\gamma} \in \gamma, \quad C_{\mathbf{K}}^{m}\left\|\mathbf{v}_{\gamma}\right\|_{0, \gamma} \leq\left\langle\mathbf{K}_{\gamma} \mathbf{v}_{\gamma}, \mathbf{v}_{\gamma}\right\rangle_{\gamma}, \quad \forall \mathbf{v}_{\gamma} L^{2}(\gamma),
\end{array}
$$

where we use the notation $(\cdot, \cdot)_{\Omega}$ and $\langle\cdot, \cdot\rangle_{\gamma}$ for the $L^{2}$ inner products on $L^{2}(\Omega)$ and $L^{2}(\gamma)$, respectively, and $\|\cdot\|_{0, \mathcal{O}}$ for the $L^{2}(\mathcal{O})$ norm on an open set $\mathcal{O} \subset$ $\mathbb{R}^{d}, d=1,2$. Here $\|\mathbf{K}(x)\|$ denotes the operator norm as does $\left\|\mathbf{K}_{\gamma}\left(x_{\gamma}\right)\right\|$. For simplicity assume that $\gamma$ is a line segment and that $\partial \gamma \subset \partial \Omega$. Flow in both $\Omega$ and $\gamma$ is assumed to be governed by Darcy's law and the law of mass conservation, and for simplicity homogeneous Dirichlet boundary conditions are imposed on 
both $\partial \gamma$ and $\partial \Omega$. Fluid exchange between $\Omega$ and $\gamma$ is through a source/sink term $\lambda$ representing the discontinuity in the flux in $\Omega$ from one side of $\gamma$ to the other. Letting $p$ and $p_{\gamma}$ represent the fluid pressure and $f$ and $f_{\gamma}$ external source terms in $\Omega$ and $\gamma$ respectively, assuming sufficient regularity of $f_{\gamma}$, we may write the equations for the model as follows:

$$
\begin{array}{ll}
\operatorname{div}(-\mathbf{K} \boldsymbol{\nabla} p)-\lambda \delta_{\gamma}=f, & \text { in } \Omega, \\
\operatorname{div}_{\tau}\left(-\mathbf{K}_{\gamma} \boldsymbol{\nabla}_{\tau} p_{\gamma}\right)+\lambda=f_{\gamma}, & \text { in } \gamma, \\
\left.p\right|_{\gamma}=p_{\gamma}, & \text { in } \gamma, \\
p=0, & \text { on } \Gamma=\partial \Omega, \\
p_{\gamma}=0, & \text { on } \partial \gamma,
\end{array}
$$

where $\delta_{\gamma}$ denotes the Dirac measure on $\gamma$, and where the operators $\operatorname{div}_{\tau}$ and $\nabla_{\tau}$ denote the derivatives in the direction obtained by rotating $\mathbf{n}_{\gamma}$ through 90 degrees. For the variational formulation, the spaces $V_{\Omega}, V_{\gamma}, \boldsymbol{V}$ and $\Lambda$ are used:

$$
V_{\Omega}=H_{0}^{1}(\Omega), \quad V_{\gamma}=H_{0}^{1}(\gamma), \quad \boldsymbol{V}=V_{\Omega} \times V_{\gamma}, \quad \text { and } \quad \Lambda=H_{00}^{-\frac{1}{2}}(\gamma)
$$

We use the same notation $\langle\cdot, \cdot\rangle_{\gamma}$ for the duality pairing between $H_{00}^{-\frac{1}{2}}(\gamma)$ and $H_{00}^{\frac{1}{2}}(\gamma)$ as that which is used for the $L^{2}(\gamma)$-inner product when the functions are sufficiently regular. Now with the bilinear form $\mathcal{A}$ on $(\boldsymbol{V} \times \Lambda)^{2}$ defined by

$$
\mathcal{A}(P, Q)=\int_{\Omega} \mathbf{K} \nabla p \cdot \nabla q+\int_{\gamma} \mathbf{K}_{\gamma} \nabla_{\tau} p_{\gamma} \cdot \nabla_{\tau} q_{\gamma}-\left\langle\lambda,\left.q\right|_{\gamma}-q_{\gamma}\right\rangle_{\gamma}+\left\langle\mu,\left.p\right|_{\gamma}-p_{\gamma}\right\rangle_{\gamma}
$$

for $P=\left(p, p_{\gamma} ; \lambda\right)$ and $Q=\left(q, q_{\gamma} ; \mu\right)$ in $\boldsymbol{V} \times \Lambda$, and the linear form $\ell$ on $\boldsymbol{V}$ defined by

$$
\ell\left(q, q_{\gamma}\right)=\int_{\Omega} f q+\int_{\gamma} f_{\gamma} q_{\gamma}
$$

for $\left(q, q_{\gamma}\right) \in \boldsymbol{V}$, the variational formulation of (1) may be written as follows:

Find $P=\left(p, p_{\gamma} ; \lambda\right) \in \boldsymbol{V} \times \Lambda$ such that

$$
\mathcal{A}(P, Q)=\ell\left(q, q_{\gamma}\right), \quad \forall Q=\left(q, q_{\gamma} ; \mu\right) \in \boldsymbol{V} \times \Lambda .
$$

In Köppel et al (2018), it was proved that (3) has a unique solution. Note that $\lambda$ can be interpreted as the jump in the flux across $\gamma: \lambda=\llbracket \mathbf{K} \nabla p \cdot \mathbf{n}_{\gamma} \rrbracket_{\gamma}$.

\section{Discretization}

Inspired by the work in Burman and Hansbo (2010a), we introduce a stabilized numerical discretization of problem (3) and show existence and uniqueness of the discrete solution as well as convergence. Recall that in Köppel et al (2018), a different primal finite element method was used to discretize (3), one that uses different discretization spaces for the Lagrange multiplier and does not have a stabilization term. With the stabilized method we do not have the minimum size constraint on the support of the Lagrange multipliers. 


\subsection{A stabilized discrete formulation}

We introduce independent finite element meshes, $\mathcal{T}_{h}$ and $\mathcal{T}_{h, \gamma}$, to define the approximation spaces $V_{h, \Omega} \subset V_{\Omega}$ and $V_{h, \gamma} \subset V_{\gamma}$. The mesh $\mathcal{T}_{h}$ on $\Omega$ is made up of triangles and/or rectangles, and $\mathcal{T}_{h, \gamma}$ is a mesh on $\gamma$. We assume that each of $\mathcal{T}_{h}$ and $\mathcal{T}_{h, \gamma}$ belongs to a uniformly regular family of discretizations. Let $h$ and $h_{\gamma}$ be the parameters associated with these families:

$$
\begin{aligned}
& h=\max _{T \in \mathcal{T}_{h}} h_{T}, \quad \text { where } h_{T}=\operatorname{diam}(T), \\
& h_{\gamma}=\max _{t \in \mathcal{T}_{h, \gamma}} h_{t}, \quad \text { where } h_{t}=\operatorname{diam}(t),
\end{aligned}
$$

For each $T \in \mathcal{T}_{h}$ let $\rho_{T}=$ the radius of the incircle of $T$, and let $\sigma_{T}=\frac{h_{T}}{\rho_{T}}$. Let $\rho_{h}=\min _{T \in \mathcal{T}_{h}} \rho_{T}$ and let $\sigma_{h}=\frac{h}{\rho_{h}}$. Let $\sigma_{\Omega}=\max _{h} \sigma_{h}$ be the upper bound guaranteed by uniform regularity.

There is naturally induced on $\gamma$ a second mesh, which we will denote $\mathcal{T}_{h, \lambda}$ (as it will be associated with the space of discrete Lagrange multipliers), that consists of the segments $T \cap \gamma$ such that $T \in \mathcal{T}_{h}$, see Fig. 1:

$$
\mathcal{T}_{h, \lambda}=\left\{s \subset \gamma: s=T \cap \gamma \text { for some } T \in \mathcal{T}_{h}\right\} .
$$

Let $\mathcal{F}_{h}$ denote the set of edges $F$ of elements $T \in \mathcal{T}_{h}$, and $\mathcal{F}_{h, \lambda}$ the set of vertices $f$ of elements $s \in \mathcal{T}_{h, \lambda}$ which do not lie on the boundary: $f \notin \partial \gamma$. The conforming approximation spaces $V_{h, \Omega}$ and $V_{h, \gamma}$ will consist of continuous

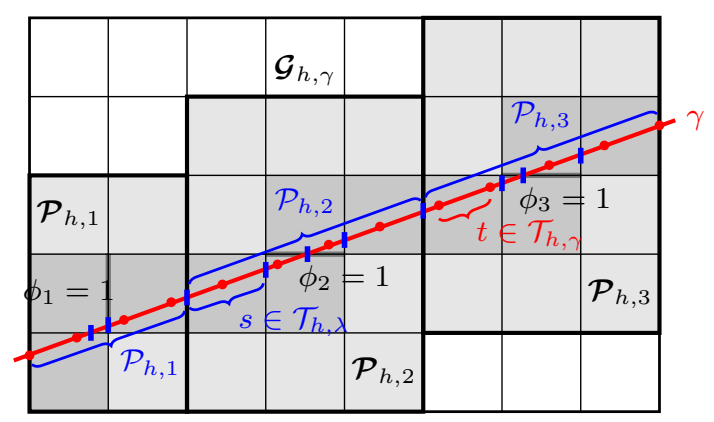

Fig. 1 Meshes $\mathcal{T}_{h}, \mathcal{T}_{h, \gamma}$ (elements $t$ delimited by red dots) and $\mathcal{T}_{h, \lambda}$ (elements $s$ delimited by blue segments). The domain $\mathcal{G}_{h, \gamma}$ around $\gamma$ is in grey. The supports of the patch elements $\mathcal{P}_{h, i}$ (in blue) and $\mathcal{P}_{h, i}$ (grey) are also depicted, with the chosen edge for $\phi_{i}$.

functions that vanish on the boundary of $\Omega$ and $\gamma$, respectively. The functions in $V_{h, \gamma}$ will be piecewise linear subordinate to the mesh $\mathcal{T}_{h, \gamma}$ while those of $V_{h, \Omega}$, subordinate to the mesh $\mathcal{T}_{h}$ will be piecewise linear or bilinear depending on whether the element is a triangle or a rectangle:

$$
\begin{aligned}
& V_{h, \Omega}=\left\{q \in H_{0}^{1}(\Omega): \forall T \in \mathcal{T}_{h},\left.q\right|_{T} \in\left\{\begin{array}{l}
\mathbb{P}^{1}(T) \text { if } T \text { is a triangle } \\
\mathbb{Q}^{1,1}(T) \text { if } T \text { is a rectangle }
\end{array}\right\},\right. \\
& V_{h, \gamma}=\left\{q_{\gamma} \in H_{0}^{1}(\gamma): \forall t \in \mathcal{T}_{h, \gamma},\left.\quad q_{\gamma}\right|_{t} \in \mathbb{P}^{1}(t)\right\}, \text { and } \boldsymbol{V}_{h}=V_{h, \Omega} \times V_{h, \gamma} .
\end{aligned}
$$


The approximation space $\Lambda_{h}$ for the Lagrange multiplier is defined as follows:

$$
\Lambda_{h}=\left\{\lambda_{h} \in L^{2}(\gamma)\left|\lambda_{h}\right|_{s} \in \mathbb{P}^{0}(s), \forall s \in \mathcal{T}_{h, \lambda}\right\}
$$

Following Burman and Hansbo (2010a) we will introduce a stabilizing term $\mathcal{J}$ in the form of a bilinear operator on $\Lambda_{h} \times \Lambda_{h}$ :

$$
\mathcal{J}\left(\lambda_{h}, \mu_{h}\right)=\sum_{f \in \mathcal{F}_{h, \lambda}} \xi h^{2} \llbracket \lambda_{h} \rrbracket f \llbracket \mu_{h} \rrbracket f,
$$

where for $\mu_{h} \in \Lambda_{h}$, and $f \in \mathcal{F}_{h, \lambda}, \llbracket \mu_{h} \rrbracket_{f}$ denotes the jump in $\mu_{h}$ across the vertex $f$ (i.e. along the fracture, and should not be confused with $\mathbb{\|} \cdot \mathbb{\|} \gamma$ which is a jump normal to $\gamma$ ). Here, for simplicity, we assume that no edge $F \in \mathcal{F}_{h}$ lies along $\gamma$ and that $\gamma$ does not contain any vertex of the mesh $\mathcal{T}_{h}$. This ensures that $\llbracket \lambda_{h} \rrbracket_{f}$ is uniquely defined when $f \in \mathcal{F}_{h, \lambda}$ and $\lambda_{h} \in \Lambda_{h}$. Otherwise defining the jump term is more cumbersome, though it poses no real problem, and in fact, some of our numerical experiments treat such cases. We remark that we will at times use the notation $\llbracket \phi \rrbracket_{f}$ for functions $\phi$ not necessarily belonging to $\Lambda_{h}$ but for which the jumps over the vertices $f \in \mathcal{F}_{h, \lambda}$ are well defined. Indeed, $\mathcal{J}(\cdot, \cdot)^{\frac{1}{2}}$ defines a semi-norm on $\Lambda_{h}$, and we have the Cauchy-Schwarz-like estimate

$$
\left|\mathcal{J}\left(\lambda_{h}, \mu_{h}\right)\right| \leq \mathcal{J}\left(\lambda_{h}, \lambda_{h}\right)^{\frac{1}{2}} \mathcal{J}\left(\mu_{h}, \mu_{h}\right)^{\frac{1}{2}} \quad \forall \lambda_{h}, \mu_{h} \in \Lambda_{h}
$$

from the usual estimate $\left|\sum_{f} a_{f} b_{f}\right| \leq\left(\sum_{f} a_{f}^{2}\right)^{\frac{1}{2}}\left(\sum_{f} b_{f}^{2}\right)^{\frac{1}{2}}$ for $a_{f}, b_{f} \in \mathbb{R}$. The formulation of the discrete stabilized problem may be written as follows:

Find $P_{h}=\left(p_{h}, p_{\gamma, h} ; \lambda_{h}\right) \in \boldsymbol{V}_{h} \times \Lambda_{h}$ such that

$$
\mathcal{A}\left(P_{h}, Q_{h}\right)+\mathcal{J}\left(\lambda_{h}, \mu_{h}\right)=\ell\left(q_{h}, q_{\gamma, h}\right), \quad \forall Q_{h}=\left(q_{h}, q_{\gamma, h} ; \mu_{h}\right) \in \boldsymbol{V}_{h} \times \Lambda_{h} .
$$

The following proposition states an approximate Galerkin orthogonality for (8) which will give the weak consistency of the method according to Burman and Hansbo (2010b).

Proposition 1 If $P$ is the solution of (3) and $P_{h}$ the solution of (8), then

$$
\mathcal{A}\left(P-P_{h}, Q_{h}\right)=\mathcal{J}\left(\lambda_{h}, \mu_{h}\right) \quad \forall Q_{h}=\left(q_{h}, q_{\gamma, h} ; \mu_{h}\right) \in \boldsymbol{V}_{h} \times \Lambda_{h} .
$$

Proof As $\boldsymbol{V}_{h} \subset \boldsymbol{V}$ and $\Lambda_{h} \subset \Lambda$, it suffices to take $Q=Q_{h} \in \boldsymbol{V}_{h} \times \Lambda_{h}$ in (3), substract (8) from (3) and use the bilinarity of $\mathcal{A}$ to obtain (9).

\subsection{Some discrete norms}

We give the definition of some norms that will be useful for obtaining the approximation properties of the space $\boldsymbol{V}_{h} \times \Lambda_{h}$. For $\zeta \in L^{2}(\gamma)$ and $h>0$ we define the discrete norms

$$
\|\zeta\|_{\frac{1}{2}, h, \gamma}^{2}=\int_{\gamma} h^{-1} \zeta^{2}=h^{-1}\|\zeta\|_{0, \gamma}^{2} \text { and }\|\zeta\|_{-\frac{1}{2}, h, \gamma}^{2}=\int_{\gamma} h \zeta^{2}=h\|\zeta\|_{0, \gamma}^{2},
$$


and we recall the associated Cauchy-Schwarz type inequality

$$
\langle\zeta, \eta\rangle_{\gamma} \leq\|\zeta\|_{-\frac{1}{2}, h, \gamma}\|\eta\|_{\frac{1}{2}, h, \gamma}, \quad \forall \zeta \text { and } \eta \in L^{2}(\gamma) .
$$

We will also use two more norms defined respectively for $Q=\left(q, q_{\gamma} ; \mu\right) \in \boldsymbol{V} \times$ $L^{2}(\gamma)$ for $Q_{h}=\left(q, q_{\gamma} ; \mu_{h}\right) \in \boldsymbol{V} \times \Lambda_{h}$, and for $h>0$ by

$$
\begin{aligned}
\|Q\|_{0, h}^{2} & :=\|\nabla q\|_{0, \Omega}^{2}+\left\|\nabla_{\tau} q_{\gamma}\right\|_{0, \gamma}^{2}+\|\mu\|_{-\frac{1}{2}, h, \gamma}^{2}+\left\|\left.q\right|_{\gamma}-q_{\gamma}\right\|_{\frac{1}{2}, h, \gamma}^{2} \\
\left\|Q_{h}\right\|_{1, h}^{2} & :=\left\|Q_{h}\right\|_{0, h}^{2}+\mathcal{J}\left(\mu_{h}, \mu_{h}\right) .
\end{aligned}
$$

That $\|\cdot\|_{0, h}$ indeed defines a norm on $\boldsymbol{V} \times L^{2}(\gamma)$ follows immediately from the Poincaré inequality. Thus $\|\cdot\|_{1, h}$ also defines a norm on $\boldsymbol{V} \times \Lambda_{h}$. That $\mathcal{A}$ is continuous in the $\|\cdot \cdot\|_{0, h}$ norm follows from the Cauchy-Schwarz inequality:

Proposition 2 There exists a constant $C_{c}$, independent of $h$, such that if $P$ and $Q$ belong to $\boldsymbol{V} \times L^{2}(\gamma)$, then

$$
\mathcal{A}(P, Q) \leq C_{c}\|P\|_{0, h}\|Q\|_{0, h} .
$$

Proof The Cauchy-Schwarz inequality, (7) and (11) yield (12) with $C_{c}=C_{\mathrm{K}}^{M}$.

\subsection{A subspace of $\Lambda_{h}$ and some approximation lemmas}

The family of inherited meshes $\mathcal{T}_{h, \lambda}$ on $\gamma$ suffers from the fact that it is not uniformly regular: while for $s \in \mathcal{T}_{h, \lambda}$, its length $h_{s} \leq h$, there is not necessarily a $\sigma_{\lambda}>0$, independent of $h$, such that $h_{s} \geq \frac{h}{\sigma_{\lambda}}$. For this reason we amalgamate elements of $\mathcal{T}_{h, \lambda}$ to obtain a supermesh $\mathcal{T}_{h, \mathcal{P}}$ of $\mathcal{T}_{h, \lambda}$ made up of patch-elements obtained by fusing two or more contiguous elements of $\mathcal{T}_{h, \lambda}$ to form $n_{h}$ pairwisedisjoint patches, $\mathcal{P}_{h, i}, i=1, \cdots n_{h}$, see Fig. 1 . The patches are used for the analysis, but are not built in practice. The patches should be constructed in such a way that the length of each patch segment is bounded above and below by a multiple of $h$; i.e. there are positive constants $c_{1}$ and $c_{2}$, independent of $h$, such that

$$
c_{1} h \leq h_{\mathcal{P}_{h, i}} \leq c_{2} h, i=1, \cdots n_{h}
$$

where $h_{\mathcal{P}_{h, i}}$ denotes the length of the patch-segment $\mathcal{P}_{h, i}$. Let $h_{\mathcal{P}}$ be the maximum value of $h_{\mathcal{P}_{h, i}}, \mathcal{P}_{h, i} \in \mathcal{T}_{h, \mathcal{P}}$. An additional constraint on the patch construction will be given in Section 3.4 following the proof of Lemma 3. From the uniform regularity of $\mathcal{T}_{h}$, the patch-segments can clearly be constructed so that the maximum number of elements $s \in \mathcal{T}_{h, \lambda}$ in a patch-element $\mathcal{P}_{h, i}$ is bounded above by some number $\bar{n}$ independent of $h$. The patches can be numbered in such a way that each of $\mathcal{P}_{h, 1}$ and $\mathcal{P}_{h, n_{h}}$ has a vertex on the boundary of $\gamma$, and such that for $i=1, \cdots, n_{h}-1, \mathcal{P}_{h, i}$ and $\mathcal{P}_{h, i+1}$ have a vertex in common. Similarly, for each $i ; i=1, \cdots, n_{h}$, the patch $\mathcal{P}_{h, i}$ contains as subsets a certain number, $n_{i}$, of cells $s_{i, \ell} \in \mathcal{T}_{h, \lambda}, \ell=1, \cdots n_{i}$ which we may assume are numbered such that the first and last cells have a vertex on $\partial \mathcal{P}_{h, i}$ and contiguous cells are 
numbered consecutively. Now define the space of patch-wise constant functions on $\gamma$

$$
\mathcal{X}_{h}=\left\{x_{h} \in L^{2}(\gamma):\left.x_{h}\right|_{\mathcal{P}_{h, i}} \in \mathbb{P}^{0}\left(\mathcal{P}_{h, i}\right), i=1, \cdots, n_{h}\right\} .
$$

(Please note that $\mathcal{X}_{h}$ is not meant to replace the multiplier space $\Lambda_{h}$ but is to be used only in the demonstrations). Then let $\pi_{p}: L^{2}(\gamma) \rightarrow \mathcal{X}_{h}$ be defined by $\eta \mapsto \pi_{\mathcal{P}} \eta$ where $\left.\pi_{\mathcal{P}} \eta\right|_{\mathcal{P}_{h, i}}=\frac{1}{h_{\mathcal{P}_{h, i}}} \int_{\mathcal{P}_{h, i}} \eta$. As $\pi_{\mathcal{P}}$ is the $L^{2}(\gamma)$-projection operator from $L^{2}(\gamma)$ onto $\mathcal{X}_{h}$, for $\mu \in L^{2}(\gamma)$,

$$
\begin{aligned}
& \left\langle\mu, \zeta_{h}\right\rangle_{\gamma}=\left\langle\pi_{\mathcal{P}} \mu, \zeta_{h}\right\rangle_{\gamma}, \quad \forall \zeta_{h} \in \mathcal{X}_{h}, \\
& \left\|\pi_{\mathcal{P}} \mu\right\|_{0, \gamma} \leq\|\mu\|_{0, \gamma}
\end{aligned}
$$

and if further $\mu \in H^{1}(\gamma)$, there is a constant $C_{\pi_{\mathcal{P}}}$, independent of $h$, such that the following Poincaré-Wirtinger-type inequality holds:

$$
\left\|\mu-\pi_{\mathcal{P}} \mu\right\|_{0, \gamma} \leq C_{\pi_{\mathcal{P}}} h_{\mathcal{P}}\left\|\nabla_{\tau} \mu\right\|_{0, \gamma}
$$

Before stating approximation lemmas we define a mesh-dependent, thickened $\gamma$ made up of the cells of $\mathcal{T}_{h}$ crossed by $\gamma$ plus an extra layer of cells on each side of $\gamma$. Let

$$
\mathcal{S}_{h}=\left\{T \in \mathcal{T}_{h}: \exists T^{\prime} \in \mathcal{T}_{h} \text { with } \bar{T} \cap \bar{T}^{\prime} \neq \emptyset \text { and } \bar{T}^{\prime} \cap \gamma \neq \emptyset\right\},
$$

and let $\mathcal{G}_{h, \gamma}$ be the interior of the union of the closures of the cells $T \in \mathcal{S}_{h}$ :

$$
\mathcal{G}_{h, \gamma}=\operatorname{Int}\left(\bigcup_{T \in \mathcal{S}_{h}} \bar{T}\right)
$$

The following two lemmas concern approximation in $\mathcal{X}_{h}$ :

Lemma 1 There exist constants $C_{1} \geq 1$ and $\tilde{C}_{1}>0$ such that for $\left(q_{h}, q_{\gamma, h}\right) \in \boldsymbol{V}_{h}$

$$
\begin{aligned}
& \left\|\left(\left.q_{h}\right|_{\gamma}-q_{\gamma, h}\right)-\pi_{\mathcal{P}}\left(\left.q_{h}\right|_{\gamma}-q_{\gamma, h}\right)\right\|_{\frac{1}{2}, h, \gamma}^{2} \leq C_{1}\left(\left\|\nabla q_{h}\right\|_{0, \mathcal{G}_{h, \gamma}}^{2}+h\left\|\nabla_{\tau} q_{\gamma, h}\right\|_{0, \gamma}^{2}\right), \\
& \tilde{C}_{1}\left\|\left.q_{h}\right|_{\gamma}-q_{\gamma, h}\right\|_{\frac{1}{2}, h, \gamma}^{2}-\left\|\nabla q_{h}\right\|_{0, \mathcal{G}_{h, \gamma}}^{2}-h\left\|\nabla_{\tau} q_{\gamma, h}\right\|_{0, \gamma}^{2} \leq\left\|\pi_{\mathcal{P}}\left(\left.q_{h}\right|_{\gamma}-q_{\gamma, h}\right)\right\|_{\frac{1}{2}, h, \gamma}^{2} .
\end{aligned}
$$

Proof The second inequality follows directly from the first with $\tilde{C}_{1}=\frac{1}{2 C_{1}}$, so we only need to prove the first. For $q_{\gamma, h}$ in $V_{h, \gamma}$ and $q_{h}$ in $V_{h, \Omega}$, we have $q_{\gamma, h} \in H^{1}(\gamma)$ and $\left.q_{h}\right|_{\gamma} \in H^{1}(\gamma)$. Thus (16) and then (13) implies that

$$
\begin{aligned}
\left\|q_{\gamma, h}-\pi_{\mathcal{P}} q_{\gamma, h}\right\|_{0, \gamma} & \leq C_{\pi_{\mathcal{P}}} c_{2} h\left\|\nabla_{\tau}\left(q_{\gamma, h}\right)\right\|_{0, \gamma} \\
\left\|\left.q_{h}\right|_{\gamma}-\pi_{\mathcal{P}}\left(\left.q_{h}\right|_{\gamma}\right)\right\|_{0, \gamma} & \leq C_{\pi_{\mathcal{P}}} c_{2} h\left\|\nabla_{\tau}\left(\left.q_{h}\right|_{\gamma}\right)\right\|_{0, \gamma}
\end{aligned}
$$

It is obvious for a grid of triangles (when $\nabla q_{h}$ is piecewise constant) but also true for a grid of rectangles that there exists $C_{\sigma}>0$ such that

$$
\left\|\nabla_{\tau}\left(\left.q_{h}\right|_{\gamma}\right)\right\|_{0, \gamma} \leq C_{\sigma} h^{-\frac{1}{2}}\left\|\nabla_{\tau} q_{h}\right\|_{0, \mathcal{G}_{h, \gamma}} \leq C_{\sigma} h^{-\frac{1}{2}}\left\|\nabla q_{h}\right\|_{0, \mathcal{G}_{h, \gamma}},
$$


where $C_{\sigma}$ depends on the uniform regularity constant $\sigma_{\Omega}$. Combining the last three inequalities we obtain

$$
\begin{aligned}
& \left\|\left(\left.q_{h}\right|_{\gamma}-q_{\gamma, h}\right)-\pi_{\mathcal{P}}\left(\left.q_{h}\right|_{\gamma}-q_{\gamma, h}\right)\right\|_{\frac{1}{2}, h, \gamma}^{2} \\
& \leq 2 h^{-1}\left(\left\|\left.q_{h}\right|_{\gamma}-\pi_{\mathcal{P}}\left(\left.q_{h}\right|_{\gamma}\right)\right\|_{0, \gamma}^{2}+\left\|q_{\gamma, h}-\pi_{\mathcal{P}} q_{\gamma, h}\right\|_{0, \gamma}^{2}\right) \\
& \leq 2 C_{\pi_{\mathcal{P}}}^{2} c_{2}^{2}\left(C_{\sigma}^{2}\left\|\nabla q_{h}\right\|_{0, \mathcal{G}_{h, \gamma}}^{2}+h\left\|\nabla_{\tau} q_{\gamma, h}\right\|_{0, \gamma}^{2}\right) .
\end{aligned}
$$

The lemma follows with $C_{1}=\max \left\{1,2 C_{\pi_{\mathcal{P}}}^{2} c_{2}^{2} \max \left\{C_{\sigma}^{2}, 1\right\}\right\}$.

Lemma 2 There is a positive constant $C_{2}$ such that if $\mu_{h} \in \Lambda_{h}$, then

$$
\left\|\mu_{h}-\pi_{\mathcal{P}} \mu_{h}\right\|_{-\frac{1}{2}, h, \gamma}^{2} \leq C_{2} \mathcal{J}\left(\mu_{h}, \mu_{h}\right) .
$$

Proof As $\pi_{\mathcal{P}}$ is an $L^{2}$-projection, it suffices to show that there is a constant $C_{2}$ such that if $\mu_{h} \in \Lambda_{h}$ there is a function $\chi_{h} \in \mathcal{X}_{h}$ such that

$$
\left\|\mu_{h}-\chi_{h}\right\|_{0, \gamma}^{2} \leq C_{2} h^{-1} \mathcal{J}\left(\mu_{h}, \mu_{h}\right) \text {. }
$$

Let $\mu_{h} \in \Lambda_{h}$. We construct $\chi_{h} \in \mathcal{X}_{h}$ such that the value $\chi_{i}$ of $\chi_{h}$ on the patch $\mathcal{P}_{h, i} \in \mathcal{T}_{h, \mathcal{P}}$ is the value $\mu_{i, 1}$ of $\mu_{h}$ on the cell $s_{i, 1} \in \mathcal{T}_{h, \lambda}$, i.e. $\chi_{i}=\mu_{i, 1}$. Then

$$
\begin{aligned}
\left\|\mu_{h}-\chi_{h}\right\|_{0, \gamma}^{2} & =\sum_{i=1}^{n_{h}}\left\|\mu_{h}-\chi_{i}\right\|_{0, \mathcal{P}_{h, i}}^{2}=\sum_{i=1}^{n_{h}} \sum_{\ell=2}^{n_{i}}\left\|\mu_{h}-\chi_{i}\right\|_{0, s_{i, \ell}}^{2} \\
& \leq h_{\lambda} \sum_{i=1}^{n_{h}} \sum_{\ell=2}^{n_{i}}\left(\mu_{i, \ell}-\mu_{i, 1}\right)^{2} \leq h \sum_{i=1}^{n_{h}} \sum_{\ell=2}^{n_{i}}\left(\sum_{\nu=1}^{\ell-1} j_{i, \nu}\right)^{2} \\
& \leq h \sum_{i=1}^{n_{h}} \sum_{\ell=2}^{n_{i}}(\ell-1) \sum_{\nu=1}^{\ell-1} j_{i, \nu}^{2} \leq h \frac{\bar{n}^{2}}{2} \sum_{i=1}^{n_{h}} \sum_{\nu=1}^{n_{i}-1} j_{i, \nu}^{2},
\end{aligned}
$$

where for $\ell=1, \cdots, n_{i} ; \mu_{i, \ell}=\left.\mu_{h}\right|_{s_{i, \ell}} ;$ for $\nu=1, \cdots, n_{i}-1 ; j_{i, \nu}=\mu_{i, \nu+1}-\mu_{i, \nu}$ and $\bar{n}$ is an upper bound, independent of $h$, on the number of cells $s \in \mathcal{T}_{h, \lambda}$ contained in a patch $\mathcal{P}_{h} \in \mathcal{T}_{h, \mathcal{P}}$. The lemma follows with $C_{2}=\frac{\bar{n}^{2}}{2 \xi}$ since

$$
\mathcal{J}\left(\mu_{h}, \mu_{h}\right)=\sum_{f \in \mathcal{F}_{h, \lambda}} \xi h^{2}\left[\mu_{h}\right]_{f}^{2} \geq \xi h^{2} \sum_{i=1}^{n_{h}} \sum_{\nu=1}^{n_{i}-1} j_{i, \nu}^{2} .
$$

\subsection{A stability estimate}

This section is devoted to the demonstration of the stability estimate for the formulation (8) given in Theorem 1. The proof relies on Lemmas 3 and 4.

Lemma 3 There exist positive constants $C_{3}, C_{4}$ and $C_{5}$ independent of $h$, such that for $\left(p_{h}, p_{\gamma, h}\right)$ in $\boldsymbol{V}_{h}$ there exists $\eta_{h} \in \Lambda_{h}$ satisfying:

$$
\begin{aligned}
& \left\langle\eta_{h},\left.p_{h}\right|_{\gamma}-p_{\gamma, h}\right\rangle_{\gamma} \geq C_{3}\left\|\pi_{\mathcal{P}}\left(\left.p_{h}\right|_{\gamma}-p_{\gamma, h}\right)\right\|_{\frac{1}{2}, h, \gamma}^{2}, \\
& \left\|\eta_{h}\right\|_{-\frac{1}{2}, h, \gamma}^{2} \leq C_{4}\left\|\pi_{\mathcal{P}}\left(\left.p_{h}\right|_{\gamma}-p_{\gamma, h}\right)\right\|_{\frac{1}{2}, h, \gamma}^{2} \\
& \mathcal{J}\left(\eta_{h}, \eta_{h}\right) \leq C_{5}\left\|\pi_{\mathcal{P}}\left(\left.p_{h}\right|_{\gamma}-p_{\gamma, h}\right)\right\|_{\frac{1}{2}, h, \gamma}^{2}
\end{aligned}
$$


Proof Given $\left(p_{h}, p_{\gamma, h}\right)$ in $\boldsymbol{V}_{h}$, define $\eta_{h} \in \mathcal{X}_{h}$ by $\eta_{h}=\frac{1}{h_{\mathcal{P}}} \pi_{\mathcal{P}}\left(\left.p_{h}\right|_{\gamma}-p_{\gamma, h}\right)$. Use equations (14) and (13) to obtain the first inequality of the lemma:

$$
\left\langle\eta_{h},\left.p_{h}\right|_{\gamma}-p_{\gamma, h}\right\rangle_{\gamma}=\left\|h_{\mathcal{P}}^{-\frac{1}{2}} \pi_{\mathcal{P}}\left(\left.p_{h}\right|_{\gamma}-p_{\gamma, h}\right)\right\|_{0, \gamma}^{2} \geq c_{2}^{-1}\left\|\pi_{\mathcal{P}}\left(\left.p_{h}\right|_{\gamma}-p_{\gamma, h}\right)\right\|_{\frac{1}{2}, h, \gamma}^{2} .
$$

For the second inequality, observe that

$$
\left\|\eta_{h}\right\|_{-\frac{1}{2}, h, \gamma}^{2}=h h_{\mathcal{P}}^{-2}\left\|\pi_{\mathcal{P}}\left(\left.p_{h}\right|_{\gamma}-p_{\gamma, h}\right)\right\|_{0, \gamma}^{2} \leq c_{1}^{-2}\left\|\pi_{\mathcal{P}}\left(\left.p_{h}\right|_{\gamma}-p_{\gamma, h}\right)\right\|_{\frac{1}{2}, h, \gamma}^{2} .
$$

For the third inequality, using the definition (6) of $\mathcal{J}$, the fact that $\eta_{h}$ is constant on patches, using (13) and the definition of $\eta_{h}$, one obtains

$$
\begin{aligned}
& \mathcal{J}\left(\eta_{h}, \eta_{h}\right)=\sum_{i=1}^{n_{h}-1} \xi h^{2}\left(\left.\eta_{h}\right|_{\mathcal{P}_{h, i}}-\left.\eta_{h}\right|_{\mathcal{P}_{h, i+1}}\right)^{2} \leq \sum_{i=1}^{n_{h}} 4 \xi h^{2}\left(\left.\eta_{h}\right|_{\mathcal{P}_{h, i}}\right)^{2} \\
& \quad \leq 4 c_{1}^{-2} \xi \sum_{i=1}^{n_{h}}\left(\left.\pi_{\mathcal{P}}\left(\left.p_{h}\right|_{\gamma}-p_{\gamma, h}\right)\right|_{\mathcal{P}_{h, i}}\right)^{2} \leq 4 c_{1}^{-3} \xi\left\|h^{-\frac{1}{2}} \pi_{\mathcal{P}}\left(\left.p_{h}\right|_{\gamma}-p_{\gamma, h}\right)\right\|_{0, \gamma}^{2}
\end{aligned}
$$

Now with $C_{3}=c_{2}^{-1}, C_{4}=c_{1}^{-2}$ and $C_{5}=4 c_{1}^{-3} \xi$, the proof is completed.

Before stating Lemma 4, still following Burman and Hansbo (2010a), we define a subspace $\mathcal{Y}_{h}$ of $V_{h, \Omega}$ consisting of certain functions having support "near" $\gamma$, (i.e. in $\mathcal{G}_{h, \gamma}$, see (17)). Toward this end we partition $\mathcal{G}_{h, \gamma}$ into a set of nonoverlapping thickened patches $\mathcal{P}_{h, i}, i=1, \cdots, n_{h}$, where $\mathcal{P}_{h, i}$ is made up of a choice of cells $T \in \mathcal{S}_{h}$ such that $\mathcal{P}_{h, i} \subset \mathcal{P}_{h, i}$, see Fig. 1. The 1D patches $\mathcal{P}_{h, i}$ should be constructed in such a way that to each thick patch $\mathcal{P}_{h, i}$, we can associate a patch function $\phi_{i} \in V_{h, \Omega}$ such that for $i=1, \cdots, n_{h}$ the patch function $\phi_{i}$ satisfies the following conditions:

$-0 \leq \phi_{i}(x) \leq 1, \quad \forall x \in \Omega$,

- $\phi_{i}$ vanishes outside $\mathcal{P}_{h, i}$,

- $\phi_{i}$ is identically equal to 1 on some edge $F \in \mathcal{F}_{h}$ cut by $\gamma$,

- there are constants $c_{3}$ and $c_{4}$ independent of $h$ and of $i$ such that

$$
c_{3} \leq \frac{1}{h} \int_{\mathcal{P}_{h, i}} \phi_{i} \quad \text { and } \quad h\left|\nabla \phi_{i}(x)\right| \leq c_{4}, \text { for a. e. } x \in \Omega .
$$

Because of the uniform regularity of $\mathcal{T}_{h}$, it is always possible to construct the patches in such a way: it suffices to amalgamate enough elements $s \in \mathcal{T}_{h, \lambda}$. The subspace $\mathcal{Y}_{h}$ is the space generated by the functions $\phi_{i} ; i=1, \cdots, n_{h}$. We note also that there is a constant $c_{5}$ independent of $h$ such that

$$
\left|\mathcal{P}_{h, i}\right| \leq c_{5} h_{\mathcal{P}_{h, i}}^{2}
$$

Lemma 4 There exist positive constants $C_{6}, C_{7}$ and $C_{8}$, independent of $h$, such that for $\lambda_{h}$ in $\Lambda_{h}$ there is an element $r_{h} \in \mathcal{Y}_{h} \subset V_{h, \Omega}$ such that:

$$
\begin{aligned}
& \left\langle\pi_{\mathcal{P}} \lambda_{h}, r_{h}\right\rangle_{\gamma} \geq C_{6}\left\|\pi_{\mathcal{P}} \lambda_{h}\right\|_{-\frac{1}{2}, h, \gamma}^{2}, \\
& \left\|\left.r_{h}\right|_{\gamma}\right\|_{\frac{1}{2}, h, \gamma}^{2} \leq C_{7}\left\|\lambda_{h}\right\|_{-\frac{1}{2}, h, \gamma}^{2} \\
& \left\|\nabla r_{h}\right\|_{0, \Omega}^{2} \leq C_{8}\left\|\lambda_{h}\right\|_{-\frac{1}{2}, h, \gamma}^{2}
\end{aligned}
$$


Proof Let $\lambda_{h} \in \Lambda_{h}$. We define $r_{h} \in \mathcal{Y}_{h} \subset V_{h, \Omega}$ by taking a linear combination of the patch functions as follows:

$$
r_{h}=\sum_{i=1}^{n_{h}} C_{\mathcal{P}_{i}} \phi_{i}, \quad \text { where } \quad C_{\mathcal{P}_{i}}=\frac{\int_{\mathcal{P}_{h, i}} h_{\mathcal{P}} \lambda_{h}}{\int_{\mathcal{P}_{h, i}} \phi_{i}} .
$$

We then have $\left.r_{h}\right|_{\Omega \backslash \mathcal{G}_{h, \gamma}}=0$, and recalling that $\phi_{i}$ vanishes outside of $\mathcal{P}_{h, i}$, we also have

$$
\int_{\mathcal{P}_{h, i}} r_{h}=\int_{\mathcal{P}_{h, i}} h_{\mathcal{P}} \lambda_{h}, \quad \forall i=1, \cdots, n_{h} .
$$

One has $\left(\int_{\mathcal{P}_{h, i}} \phi_{i}\right)^{-2} \leq c_{3}^{-2} h^{-2}$ from (18), and $\left(\int_{\mathcal{P}_{h, i}} \lambda_{h}\right)^{2} \leq h_{\mathcal{P}_{h, i}} \int_{\mathcal{P}_{h, i}} \lambda_{h}^{2}$ by the Cauchy-Schwarz inequality. Thus, using (13), we have the estimate

$$
C_{\mathcal{P}_{i}}^{2} \leq c_{2}^{3} c_{3}^{-2} \int_{\mathcal{P}_{h, i}} h \lambda_{h}^{2} \quad \forall i=1, \cdots, n_{h} .
$$

Now to check that $r_{h}$ satisfies the first inequality of the lemma, since $\pi_{P} \lambda_{h}$ is constant on $\mathcal{P}_{h, i}, i=1, \cdots, n_{h}$, we can use (21), (14) and then (13) to obtain

$$
\begin{aligned}
\left\langle\pi_{\mathcal{P}} \lambda_{h}, r_{h}\right\rangle_{\gamma} & =\left.\sum_{i=1}^{n_{h}}\left(\pi_{\mathcal{P}} \lambda_{h}\right)\right|_{\mathcal{P}_{h, i}} \int_{\mathcal{P}_{h, i}} r_{h}=\left.\sum_{i=1}^{n_{h}}\left(\pi_{\mathcal{P}} \lambda_{h}\right)\right|_{\mathcal{P}_{h, i}} \int_{\mathcal{P}_{h, i}} h_{\mathcal{P}} \lambda_{h} \\
& =h_{\mathcal{P}}\left\langle\pi_{\mathcal{P}} \lambda_{h}, \lambda_{h}\right\rangle_{\gamma}=h_{\mathcal{P}} \int_{\gamma}\left(\pi_{\mathcal{P}} \lambda_{h}\right)^{2} \geq c_{1}\left\|\pi_{\mathcal{P}} \lambda_{h}\right\|_{-\frac{1}{2}, h, \gamma}^{2} .
\end{aligned}
$$

For the second inequality we note that by the definition of $r_{h}$ in (20), we have

$$
\left\|\left.r_{h}\right|_{\gamma}\right\|_{\frac{1}{2}, h, \gamma}^{2}=h^{-1} \sum_{i=1}^{n_{h}} C_{\mathcal{P}_{i}}^{2} \int_{\mathcal{P}_{h, i}} \phi_{i}^{2} \leq c_{2} \sum_{i=1}^{n_{h}} C_{\mathcal{P}_{i}}^{2}
$$

because (13), together with the fact that $\left|\phi_{i}(x)\right| \leq 1, \forall x \in \gamma$, implies that $\int_{\mathcal{P}_{h, i}} \phi_{i}^{2} \leq h_{\mathcal{P}_{h, i}} \leq c_{2} h$. Thus using (22), one obtains

$$
\left\|\left.r_{h}\right|_{\gamma}\right\|_{\frac{1}{2}, h, \gamma}^{2} \leq \sum_{i=1}^{n_{h}} c_{2}^{4} c_{3}^{-2} \int_{\mathcal{P}_{h, i}} h \lambda_{h}^{2}=c_{2}^{4} c_{3}^{-2}\left\|\lambda_{h}\right\|_{-\frac{1}{2}, h, \gamma} .
$$

For the third inequality, since the supports of the $\phi_{i}$ 's are disjoint, we have

$$
\left\|\boldsymbol{\nabla} r_{h}\right\|_{0, \Omega}^{2}=\sum_{i=1}^{n_{h}} C_{\mathcal{P}_{i}}^{2} \int_{\mathcal{P}_{h, i}}\left(\boldsymbol{\nabla} \phi_{i}\right)^{2} \leq c_{2}^{2} c_{4}^{2} c_{5} \sum_{i=1}^{n_{h}} C_{\mathcal{P}_{i}}^{2} \leq c_{2}^{5} c_{3}^{-2} c_{4}^{2} c_{5} \sum_{i=1}^{n_{h}} \int_{\mathcal{P}_{h, i}} h \lambda_{h}^{2},
$$

where we have used (18), then (19) and (22). The lemma now follows with $C_{6}=c_{1}, C_{7}=c_{2}^{4} c_{3}^{-2}$ and $C_{8}=c_{2}^{5} c_{3}^{-2} c_{4}^{2} c_{5}$.

We can now state a stability theorem for the problem given by (8). 
Theorem 1 Let $\xi>0$. There is a positive constant $\theta$, independent of $h$, such that if $P_{h}=\left(p_{h}, p_{\gamma, h} ; \lambda_{h}\right) \in \boldsymbol{V}_{h} \times \Lambda_{h}$, then

$$
\theta\left\|P_{h}\right\|_{1, h} \leq \sup _{\substack{Q_{h}=\left(q_{h}, q_{\gamma, h} ; \mu_{h}\right) \\ Q_{h} \in V_{h} \times \lambda_{h}}} \frac{\mathcal{A}\left(P_{h}, Q_{h}\right)+\mathcal{J}\left(\lambda_{h}, \mu_{h}\right)}{\left\|Q_{h}\right\|_{1, h}} .
$$

Proof Clearly, it suffices to show that there exist positive constants $\theta_{1}$ and $\theta_{2}$ such that if $P_{h}=\left(p_{h}, p_{\gamma, h}, \lambda_{h}\right) \in \boldsymbol{V}_{h} \times \Lambda_{h}$ there exists $Q_{h}=\left(q_{h}, q_{\gamma, h}, \mu_{h}\right) \in$ $\boldsymbol{V}_{h} \times \Lambda_{h}$ such that

$$
\theta_{1}\left\|Q_{h}\right\|_{1, h} \leq\left\|P_{h}\right\|_{1, h} \quad \text { and } \quad \theta_{2}\left\|P_{h}\right\|_{1, h}^{2} \leq \mathcal{A}\left(P_{h}, Q_{h}\right)+\mathcal{J}\left(\lambda_{h}, \mu_{h}\right) .
$$

Let $P_{h}=\left(p_{h}, p_{\gamma, h} ; \lambda_{h}\right) \in \boldsymbol{V}_{h} \times \Lambda_{h}$. The idea is to put

$$
Q_{h}=P_{h}+R_{h}, \quad \text { with } R_{h}=\left(-c_{r} r_{h}, 0 ; c_{\eta} \eta_{h}\right) \in V_{h} \times \Lambda_{h}
$$

with $\eta_{h}$ and $r_{h}$ as constructed in Lemmas (3) and (4), respectively, and with $c_{r}$ and $c_{\eta}$ positive constants to be determined in such a way that both equations of (24) are satisfied with $\theta_{1}$ and $\theta_{2}$ independent of the choice of $P_{h}$.

To obtain the first estimate of (24), we use Lemmas 3 and 4 with (15):

$$
\begin{gathered}
\left\|Q_{h}\right\|_{1, h}^{2}=\left\|\nabla p_{h}-c_{r} \nabla r_{h}\right\|_{0, \Omega}^{2}+\left\|\nabla_{\tau} p_{\gamma, h}\right\|_{0, \gamma}^{2}+\left\|\lambda_{h}+c_{\eta} \eta_{h}\right\|_{-\frac{1}{2}, h, \gamma}^{2} \\
+\left\|\left.p_{h}\right|_{\gamma}-\left.c_{r} r_{h}\right|_{\gamma}-p_{\gamma, h}\right\|_{\frac{1}{2}, h, \gamma}^{2}+\mathcal{J}\left(\lambda_{h}+c_{\eta} \eta_{h}, \lambda_{h}+c_{\eta} \eta_{h}\right) \\
\leq 2\left(\left\|P_{h}\right\|_{1, h}^{2}+c_{\eta}^{2}\left(\left\|\eta_{h}\right\|_{-\frac{1}{2}, h, \gamma}^{2}+\mathcal{J}\left(\eta_{h}, \eta_{h}\right)\right)+c_{r}^{2}\left(\left\|\nabla r_{h}\right\|_{0, \Omega}^{2}+\left\|r_{h}\right\|_{\frac{1}{2}, h, \gamma}^{2}\right)\right) \\
\leq 2\left(\left\|P_{h}\right\|_{1, h}^{2}+c_{\eta}^{2}\left(C_{4}+C_{5}\right)\left\|\left(\left.p_{h}\right|_{\gamma}-p_{\gamma, h}\right)\right\|_{\frac{1}{2}, h, \gamma}^{2}+c_{r}^{2}\left(C_{7}+C_{8}\right)\left\|\lambda_{h}\right\|_{-\frac{1}{2}, h, \gamma}^{2}\right) .
\end{gathered}
$$

Thus putting $\theta_{1}=\left(2\left(1+\max \left\{c_{\eta}^{2}\left(C_{4}+C_{5}\right), c_{r}^{2}\left(C_{7}+C_{8}\right)\right\}\right)\right)^{-\frac{1}{2}}$ we obtain the first inequality of (24).

For the second estimate of (24), letting $\mu_{h}=\lambda_{h}+c_{\eta} \eta_{h}$, we have

$$
\mathcal{A}\left(P_{h}, Q_{h}\right)+\mathcal{J}\left(\lambda_{h}, \mu_{h}\right)=\mathcal{A}\left(P_{h}, P_{h}\right)+\mathcal{J}\left(\lambda_{h}, \lambda_{h}\right)+\mathcal{A}\left(P_{h}, R_{h}\right)+c_{\eta} \mathcal{J}\left(\lambda_{h}, \eta_{h}\right) .
$$

Now we bound from below each term in (25). For the first two terms,

$$
\begin{aligned}
\mathcal{A}\left(P_{h}, P_{h}\right)+\mathcal{J}\left(\lambda_{h}, \lambda_{h}\right) & =\left\|\mathbf{K}^{\frac{1}{2}} \boldsymbol{\nabla} p_{h}\right\|_{0, \Omega}^{2}+\left\|\mathbf{K}_{\gamma}^{\frac{1}{2}} \boldsymbol{\nabla}_{\tau} p_{\gamma, h}\right\|_{0, \gamma}^{2}+\mathcal{J}\left(\lambda_{h}, \lambda_{h}\right) \\
& \geq C_{\mathbf{K}}^{m}\left(\left\|\nabla p_{h}\right\|_{0, \Omega}^{2}+\left\|\nabla_{\tau} p_{\gamma, h}\right\|_{0, \gamma}^{2}\right)+\mathcal{J}\left(\lambda_{h}, \lambda_{h}\right) .
\end{aligned}
$$

For the fourth term, using (7), Young's inequality, then the third inequality of Lemma 3 and (15), we obtain, for each $\varepsilon_{\eta}>0$,

$$
\begin{aligned}
c_{\eta} \mathcal{J}\left(\lambda_{h}, \eta_{h}\right) & \geq-c_{\eta} \mathcal{J}\left(\lambda_{h}, \lambda_{h}\right)^{\frac{1}{2}} \mathcal{J}\left(\eta_{h}, \eta_{h}\right)^{\frac{1}{2}} \geq-\frac{c_{\eta}}{\varepsilon_{\eta}} \mathcal{J}\left(\lambda_{h}, \lambda_{h}\right)-\frac{c_{\eta} \varepsilon_{\eta}}{4} \mathcal{J}\left(\eta_{h}, \eta_{h}\right) \\
& \geq-\frac{c_{\eta}}{\varepsilon_{\eta}} \mathcal{J}\left(\lambda_{h}, \lambda_{h}\right)-\frac{c_{\eta} \varepsilon_{\eta}}{4} C_{5}\left\|\left.p_{h}\right|_{\gamma}-p_{\gamma, h}\right\|_{\frac{1}{2}, h, \gamma}^{2} .
\end{aligned}
$$


There remains to bound the third term in (25):

$$
\mathcal{A}\left(P_{h}, R_{h}\right)=-c_{r} \int_{\Omega} \mathbf{K} \nabla p_{h} \cdot \nabla r_{h}+c_{r}\left\langle\lambda_{h},\left.r_{h}\right|_{\gamma}\right\rangle_{\gamma}+c_{\eta}\left\langle\eta_{h},\left.p_{h}\right|_{\gamma}-p_{\gamma, h}\right\rangle_{\gamma} .
$$

For the first term of the right hand side of (28) we have, for each $\varepsilon_{r}>0$,

$$
\begin{aligned}
-c_{r} \int_{\Omega} \mathbf{K} \nabla p_{h} \cdot \nabla r_{h} & \geq-\frac{c_{r}}{\varepsilon_{r}}\left\|\mathbf{K}^{\frac{1}{2}} \nabla p_{h}\right\|_{\Omega}^{2}-\frac{c_{r} \varepsilon_{r}}{4}\left\|\mathbf{K}^{\frac{1}{2}} \nabla r_{h}\right\|_{\Omega}^{2} \\
& \geq-\frac{c_{r}}{\varepsilon_{r}} C_{\mathbf{K}}^{M}\left\|\nabla p_{h}\right\|_{\Omega}^{2}-\frac{c_{r} \varepsilon_{r}}{4} C_{\mathbf{K}}^{M} C_{8}\left\|\lambda_{h}\right\|_{-\frac{1}{2}, h, \gamma}^{2}
\end{aligned}
$$

For the second term, using the Cauchy-Schwarz type inequality (11), the first inequality of Lemma 4, Young's inequality, the second inequality of Lemma 4 and Lemma 2, we obtain

$$
\begin{aligned}
c_{r}\left\langle\lambda_{h},\left.r_{h}\right|_{\gamma}\right\rangle_{\gamma}= & c_{r}\left\langle\lambda_{h}-\pi_{\mathcal{P}} \lambda_{h},\left.r_{h}\right|_{\gamma}\right\rangle_{\gamma}+c_{r}\left\langle\pi_{\mathcal{P}} \lambda_{h},\left.r_{h}\right|_{\gamma}\right\rangle_{\gamma} \\
\geq & -\frac{c_{r}}{\varepsilon_{r}}\left\|\lambda_{h}-\pi_{\mathcal{P}} \lambda_{h}\right\|_{-\frac{1}{2}, h, \gamma}^{2}-\frac{c_{r} \varepsilon_{r}}{4}\left\|\left.r_{h}\right|_{\gamma}\right\|_{\frac{1}{2}, h, \gamma}^{2}+c_{r} C_{6}\left\|\pi_{\mathcal{P}} \lambda_{h}\right\|_{-\frac{1}{2}, h, \gamma}^{2} \\
\geq & -\frac{c_{r}}{\varepsilon_{r}}\left\|\lambda_{h}-\pi_{\mathcal{P}} \lambda_{h}\right\|_{-\frac{1}{2}, h, \gamma}^{2}-\frac{c_{r} \varepsilon_{r}}{4} C_{7}\left\|\lambda_{h}\right\|_{-\frac{1}{2}, h, \gamma}^{2} \\
& +c_{r} C_{6}\left(\frac{1}{2}\left\|\lambda_{h}\right\|_{-\frac{1}{2}, h, \gamma}^{2}-\left\|\lambda_{h}-\pi_{\mathcal{P}} \lambda_{h}\right\|_{-\frac{1}{2}, h, \gamma}^{2}\right) \\
\geq & -c_{r}\left(C_{6}+\frac{1}{\varepsilon_{r}}\right)\left\|\lambda_{h}-\pi_{\mathcal{P}} \lambda_{h}\right\|_{-\frac{1}{2}, h, \gamma}^{2}+c_{r}\left(\frac{1}{2} C_{6}-\frac{\varepsilon_{r}}{4} C_{7}\right)\left\|\lambda_{h}\right\|_{-\frac{1}{2}, h, \gamma}^{2} \\
\geq & -c_{r}\left(C_{6}+\frac{1}{\varepsilon_{r}}\right) C_{2} \mathcal{J}\left(\lambda_{h}, \lambda_{h}\right)+c_{r}\left(\frac{1}{2} C_{6}-\frac{\varepsilon_{r}}{4} C_{7}\right)\left\|\lambda_{h}\right\|_{-\frac{1}{2}, h, \gamma}^{2},
\end{aligned}
$$

where we have also used the inequality $\|a\|^{2} \leq 2\left(\|a-b\|^{2}+\|b\|^{2}\right)$ which gives $\|b\|^{2} \geq \frac{1}{2}\|a\|^{2}-\|a-b\|^{2}$. For the third term of the right hand side of (28) we use the first inequality of Lemma 3 and then Lemma 1 to obtain

$$
\begin{aligned}
& c_{\eta}\left\langle\eta_{h},\left.p_{h}\right|_{\gamma}-p_{\gamma, h}\right\rangle_{\gamma} \geq c_{\eta} C_{3}\left\|\pi_{\mathcal{P}}\left(\left.p_{h}\right|_{\gamma}-p_{\gamma, h}\right)\right\|_{\frac{1}{2}, h, \gamma}^{2} \\
& \quad \geq c_{\eta} C_{3}\left(\tilde{C}_{1}\left\|\left.p_{h}\right|_{\gamma}-p_{\gamma, h}\right\|_{\frac{1}{2}, h, \gamma}^{2}-\left\|\nabla p_{h}\right\|_{0, \mathcal{G}_{h, \gamma}}^{2}-h\left\|\nabla_{\tau} p_{\gamma, h}\right\|_{0, \gamma}^{2}\right) .
\end{aligned}
$$

Thus adding equations (26), (27), (29), (30) and (31), we have

$$
\begin{aligned}
& \mathcal{A}\left(P_{h}, Q_{h}\right)+\mathcal{J}\left(\lambda_{h}, \mu_{h}\right) \geq\left(C_{\mathbf{K}}^{m}-c_{\eta} C_{3}-\frac{c_{r}}{\varepsilon_{r}} C_{\mathbf{K}}^{M}\right)\left\|\nabla p_{h}\right\|_{0, \Omega}^{2} \\
& \quad+\left(C_{\mathbf{K}}^{m}-c_{\eta} C_{3} h\right)\left\|\nabla_{\tau} p_{\gamma, h}\right\|_{0, \gamma}^{2}+c_{\eta}\left(\tilde{C}_{1} C_{3}-\frac{\varepsilon_{\eta}}{4} C_{5}\right)\left\|\left.p_{h}\right|_{\gamma}-p_{\gamma, h}\right\|_{\frac{1}{2}, h, \gamma}^{2} \\
& \quad+c_{r}\left(\frac{C_{6}}{2}-\frac{\varepsilon_{r}}{4}\left(C_{7}+C_{\mathbf{K}}^{M} C_{8}\right)\right)\left\|\lambda_{h}\right\|_{-\frac{1}{2}, h, \gamma}^{2}+\left(1-\frac{c_{\eta}}{\varepsilon_{\eta}}-c_{r} C_{2}\left(C_{6}+\frac{1}{\varepsilon_{r}}\right)\right) \mathcal{J}\left(\lambda_{h}, \lambda_{h}\right) .
\end{aligned}
$$

To complete the demonstration of the second estimate in (24), we have only to choose $\varepsilon_{\eta}, \varepsilon_{r}, c_{r}$ and $c_{\eta}$ such that all of the constant factors above are positive. This can be done by choosing, for instance, $\varepsilon_{r}=\frac{C_{6}}{C_{7}+C_{\mathrm{K}}^{M} C_{8}}, \varepsilon_{\eta}=\frac{2 \tilde{C}_{1} C_{3}}{C_{5}}, c_{r}=$ $\min \left\{\frac{1}{4 C_{2}\left(C_{6}+\frac{1}{\varepsilon_{r}}\right)}, \frac{\varepsilon_{r}}{4} \frac{C_{\mathrm{K}}^{m}}{C_{\mathbf{K}}^{M}}\right\}$ and $c_{\eta}=\min \left\{\frac{C_{\mathrm{K}}^{m}}{2 h C_{3}}, \frac{C_{\mathrm{K}}^{m}}{4 C_{3}}, \frac{\varepsilon_{\eta}}{4}\right\}$. We can then set $\theta_{2}=$ $\min \left\{\frac{1}{2}, \frac{1}{2} C_{\mathbf{K}}^{m}, \frac{1}{2} c_{\eta} \tilde{C}_{1} C_{3}, \frac{1}{4} c_{r} C_{6}\right\}$.

From Theorem 1, we deduce the following corollary.

Corollary 1 For any $\xi>0$, formulation (8) admits a unique solution $P_{h}=$ $\left(p_{h}, p_{\gamma, h} ; \lambda_{h}\right) \in \boldsymbol{V}_{h} \times \Lambda_{h}$. 


\subsection{Convergence}

Here we prove a Céa-type, best-approximation result and consider the question of convergence rates. The convergence depends on approximation results that are derived under assumptions concerning the regularity of problem (3).

Proposition 3 Let $P=\left(p, p_{\gamma} ; \lambda\right) \in \boldsymbol{V} \times \Lambda$ be the solution of (3) and let $P_{h}=$ $\left(p_{h}, p_{\gamma, h} ; \lambda_{h}\right) \in \boldsymbol{V}_{h} \times \Lambda_{h}$ be the solution of (8). Then there is a constant $\theta_{c}>0$ independent of $h$ such that for each $Q_{h}=\left(q_{h}, q_{\gamma, h} ; \mu_{h}\right) \in \boldsymbol{V}_{h} \times \Lambda_{h}$,

$$
\left\|P-P_{h}\right\|_{0, h}+\mathcal{J}\left(\lambda_{h}, \lambda_{h}\right)^{\frac{1}{2}} \leq \theta_{c}\left(\left\|P-Q_{h}\right\|_{0, h}+\mathcal{J}\left(\mu_{h}, \mu_{h}\right)^{\frac{1}{2}}\right) .
$$

Proof Let $Q_{h}=\left(q_{h}, q_{\gamma, h} ; \mu_{h}\right) \in \boldsymbol{V}_{h} \times \Lambda_{h}$. From the triangle inequality we have

$$
\left\|P-P_{h}\right\|_{0, h}+\mathcal{J}\left(\lambda_{h}, \lambda_{h}\right)^{\frac{1}{2}} \leq\left\|P-Q_{h}\right\|_{0, h}+\mathcal{J}\left(\mu_{h}, \mu_{h}\right)^{\frac{1}{2}}+\sqrt{2}\left\|Q_{h}-P_{h}\right\|_{1, h} .
$$

Because of (23) and (9), there exists $R_{h}=\left(r_{h}, r_{h, \gamma} ; \eta_{h}\right) \in \boldsymbol{V}_{h} \times \Lambda_{h}$ with

$$
\begin{aligned}
\theta\left\|Q_{h}-P_{h}\right\|_{1, h} & \leq \frac{\mathcal{A}\left(Q_{h}-P_{h}, R_{h}\right)+\mathcal{J}\left(\mu_{h}-\lambda_{h}, \eta_{h}\right)}{\left\|R_{h}\right\|_{1, h}} \\
& =\frac{\mathcal{A}\left(Q_{h}-P, R_{h}\right)+\mathcal{J}\left(\mu_{h}, \eta_{h}\right)}{\left\|R_{h}\right\|_{1, h}} .
\end{aligned}
$$

Thus using the continuity (12) and inequality (7), we obtain

$$
\begin{aligned}
\theta\left\|Q_{h}-P_{h}\right\|_{1, h} & \leq C_{c}\left\|Q_{h}-P\right\|_{0, h} \frac{\left\|R_{h}\right\|_{0, h}}{\left\|R_{h}\right\|_{1, h}}+\mathcal{J}\left(\mu_{h}, \mu_{h}\right)^{\frac{1}{2}} \frac{\mathcal{J}\left(\eta_{h}, \eta_{h}\right)^{\frac{1}{2}}}{\left\|R_{h}\right\|_{1, h}} \\
& \leq C_{c}\left\|Q_{h}-P\right\|_{0, h}+\mathcal{J}\left(\mu_{h}, \mu_{h}\right)^{\frac{1}{2}} .
\end{aligned}
$$

Then (32) follows from (33) and (34) with $\theta_{c}=\max \left\{1+\frac{\sqrt{2} C_{c}}{\theta}, 1+\frac{\sqrt{2}}{\theta}\right\}$.

We now recall two trace inequalities. The proof of the first may be found in (Costabel, 1988, Lemma 3.6) (cf. also (Ding, 1996, Theorem 1)). For $\mathcal{O}$ a Lipschitz domain, and $\alpha \in\left(\frac{1}{2}, \frac{3}{2}\right)$, there is a constant $\widetilde{C}_{t r}$ such that

$$
\left\|\left.\phi\right|_{\partial \mathcal{O}}\right\|_{\alpha-\frac{1}{2}, \partial \mathcal{O}} \leq \widetilde{C}_{t r}\|\phi\|_{\alpha, \mathcal{O}} \quad \forall \phi \in H^{\alpha}(\mathcal{O}) .
$$

The second is a multiplicative trace inequality which follows from (Girault and Glowinski, 1995, Lemma 2), or from Ainsworth (2007), see also (Köppel et al, 2018, Lemma 1). There is a constant $C_{t r}>0$ such that if $T$ a triangle or rectangle

$$
\|q\|_{0, \gamma \cap T}^{2} \leq C_{t r}\left(h^{-1}\|q\|_{0, T}^{2}+h\|\nabla q\|_{0, T}^{2}\right), \quad \forall q \in H^{1}(T) .
$$

For approximation results we will use the Scott-Zhang interpolation operators associated with the approximation spaces $V_{h, \Omega}$ and $V_{h, \gamma}$ :

$$
\mathcal{I}_{h}^{\Omega}: H_{0}^{1}(\Omega) \longrightarrow V_{h, \Omega} \quad \text { and } \quad \mathcal{I}_{h}^{\gamma}: H_{0}^{1}(\gamma) \longrightarrow V_{h, \gamma} .
$$


We have that if $\frac{1}{2}<\alpha \leq 2$ and $0 \leq \beta \leq \alpha$, then

$$
\begin{array}{ll}
\left\|\mathcal{I}_{h}^{\Omega} q-q\right\|_{\beta, \Omega} \leq C_{\mathcal{I}_{\Omega}} h^{\alpha-\beta}|q|_{\alpha, \Omega}, & \forall q \in H^{\alpha}(\Omega), \\
\left\|\mathcal{I}_{h}^{\gamma} q_{\gamma}-q_{\gamma}\right\|_{\beta, \gamma} \leq C_{\mathcal{I}_{\gamma}} h_{\gamma}^{\alpha-\beta}\left|q_{\gamma}\right|_{\alpha, \gamma}, & \forall q_{\gamma} \in H^{\alpha}(\gamma),
\end{array}
$$

where $|\cdot|_{\alpha}$ is an $\alpha$-semi-norm; see (Ern and Guermond, 2004, Section 1.6.2).

Define $\pi_{h}$ to be the $L^{2}(\Omega)$-projection onto $\mathbb{P}^{0}\left(\mathcal{T}_{h}\right)$, the space of piecewise constant functions on $\Omega$ subordinate to $\mathcal{T}_{h}$. We have

$$
\left\|\pi_{h} q-q\right\|_{0, \Omega} \leq C_{\pi_{h}} h\|\nabla q\|_{0, \Omega}, \quad \forall q \in H^{1}(\Omega) .
$$

We will make use of the following auxiliary problem: let $\Omega_{1}$ and $\Omega_{2}$ be the two subdomains of $\Omega$ obtained by splitting $\Omega$ along $\gamma$. For $\zeta \in H^{\frac{1}{2}}(\gamma)$ and for $j=1,2$, let $r_{\zeta, j} \in H^{1}\left(\Omega_{j}\right)$ be the solution of

$$
\begin{array}{ll}
-\Delta r_{\zeta, j}=0 & \text { in } \Omega_{j}, \\
r_{\zeta, j}=\zeta & \text { on } \gamma, \\
\nabla r_{\zeta, j} \cdot \mathbf{n}=0 & \text { on } \partial \Omega_{j} \backslash \gamma
\end{array}
$$

These problems are well posed $\forall \zeta \in H^{\frac{1}{2}}(\gamma)$ and for $j=1,2$, (cf. (Galvis and Sarkis, 2007, Lemma 2.1)) and $r_{\zeta}$, defined by $\left.r_{\zeta}\right|_{\Omega_{j}}=r_{\zeta, j}$, belongs to $H^{1}(\Omega)$ as $r_{\zeta, 1}$ and $r_{\zeta, 2}$ coincide on $\gamma$. We have

$$
\left\|r_{\zeta}\right\|_{1, \Omega} \leq C_{a}\|\zeta\|_{\frac{1}{2}, \gamma}
$$

Lemma 5 There exists a constant $C_{9}$ such that if $\zeta \in H^{\frac{1}{2}}(\gamma)$, and $r_{\zeta} \in H^{1}(\Omega)$ is such that $\left.r_{\zeta}\right|_{\Omega_{1}}$ and $\left.r_{\zeta}\right|_{\Omega_{2}}$ are the solutions to (39), for $j=1$ and 2 , then

$$
\left\|\zeta-\left.\left(\pi_{h} r_{\zeta}\right)\right|_{\gamma}\right\|_{-\frac{1}{2}, h, \gamma} \leq C_{9} h\|\zeta\|_{\frac{1}{2}, \gamma} \quad \text { and } \quad \mathcal{J}\left(\left.\left(\pi_{h} r_{\zeta}\right)\right|_{\gamma},\left.\left(\pi_{h} r_{\zeta}\right)\right|_{\gamma}\right)^{\frac{1}{2}} \leq C_{9} h\|\zeta\|_{\frac{1}{2}, \gamma} .
$$

Proof Both of these estimates are based on the trace inequality (36) applied cell by cell. For the first estimate, inequality (36) is applied for each cell $T \in \mathcal{T}_{h}$ cut by $\gamma$, to $\gamma \cap T$ in relation to $T$ : because $\zeta$ and $r_{\zeta}$ agree on $\gamma$ and because $\left.r_{\zeta}\right|_{T}$ and $\left.\left(\pi_{h} r_{\zeta}\right)\right|_{T}$ belong to $H^{1}(T)$, using (10) and then using (36) for each cell cut by $\gamma$ and summing over these cells and finally applying (38), we obtain

$$
\begin{aligned}
& \left\|\zeta-\left.\left(\pi_{h} r_{\zeta}\right)\right|_{\gamma}\right\|_{-\frac{1}{2}, h, \gamma}^{2}=h\left\|\left.\left(r_{\zeta}-\pi_{h} r_{\zeta}\right)\right|_{\gamma}\right\|_{0, \gamma}^{2} \\
& \leq C_{t r}\left(\left\|r_{\zeta}-\pi_{h} r_{\zeta}\right\|_{0, \mathcal{G}_{h, \gamma}}^{2}+h^{2}\left\|\nabla r_{\zeta}\right\|_{0, \mathcal{G}_{h, \gamma}}^{2}\right) \\
& \leq C_{t r}\left(1+C_{\pi_{h}}^{2}\right) h^{2}\left\|\nabla r_{\zeta}\right\|_{0, \mathcal{G}_{h, \gamma}}^{2} .
\end{aligned}
$$

The first estimate, with $C_{9}=\left(C_{t r}\left(1+C_{\pi_{h}}^{2}\right)\right)^{\frac{1}{2}} C_{a}$, now follows from (40).

For the second estimate, inequality (36) is applied for each edge $F \in \mathcal{F}_{h}$ cut by $\gamma$, to $F$ in relation to each cell $T \in \mathcal{T}_{h}$ having $F$ as an edge: using the 
definition of $\mathcal{J}$, the fact that, for each edge $F \in \mathcal{F}_{h}, \llbracket \pi_{h} r_{\zeta} \rrbracket_{F}$ is constant and $\llbracket r_{\zeta} \rrbracket_{F}=0$ and $h \leq \frac{|F|}{\rho_{h}} h \leq \sigma_{\Omega}|F|$ and then using (36), (38) and (40) we obtain

$$
\begin{aligned}
\mathcal{J}\left(\left.\left(\pi_{h} r_{\zeta}\right)\right|_{\gamma},\left.\left(\pi_{h} r_{\zeta}\right)\right|_{\gamma}\right) & =\left.\sum_{f \in \mathcal{F}_{h, \lambda}} \xi h^{2} \llbracket\left(\pi_{h} r_{\zeta}\right)\right|_{\gamma} \rrbracket_{f}^{2} \leq \xi \sigma_{\Omega} h \sum_{f \in \mathcal{F}_{h, \lambda}} \int_{F_{f}} \llbracket \pi_{h} r_{\zeta} \rrbracket_{F_{f}}^{2} \\
& =\xi \sigma_{\Omega} h \sum_{f \in \mathcal{F}_{h, \lambda}}\left\|\llbracket r_{\zeta}-\pi_{h} r_{\zeta} \rrbracket_{F_{f}}\right\|_{0, F_{f}}^{2} \\
& \leq 2 \xi \sigma_{\Omega} \sum_{f \in \mathcal{F}_{h, \lambda}} \sum_{i=1}^{2} C_{t r}\left(\left\|r_{\zeta}-\pi_{h} r_{\zeta}\right\|_{0, T_{F_{f}}^{i}}^{2}+h^{2}\left\|\nabla r_{\zeta}\right\|_{0, T_{F_{f}}^{i}}^{2}\right) \\
& \leq 2 \xi \sigma_{\Omega} \sum_{f \in \mathcal{F}_{h, \lambda}} \sum_{i=1}^{2} C_{t r}\left(1+C_{\pi_{h}}^{2}\right) h^{2}\left\|\nabla r_{\zeta}\right\|_{0, T_{F_{f}}^{i}}^{2} \\
& \leq 4 \xi \sigma_{\Omega} C_{t r}\left(1+C_{\pi_{h}}^{2}\right) h^{2}\left\|\nabla r_{\zeta}\right\|_{0, \Omega}^{2}
\end{aligned}
$$

where for $f \in \mathcal{F}_{h, \lambda}, F_{f}$ denotes the edge in $\mathcal{F}_{h}$ containing $f$, and $T_{F_{f}}^{1}$ and $T_{F_{f}}^{2}$ are the two cells in $\mathcal{T}_{h}$ having $F_{f}$ as an edge. The second estimate now follows as above with $C_{9}=2\left(\xi \sigma_{\Omega} C_{t r}\left(1+C_{\pi_{h}}^{2}\right)\right)^{\frac{1}{2}} C_{a}$.

Lemma 6 Assume that $p \in H^{1+\alpha}(\Omega)$ and $\left.p\right|_{\Omega_{j}} \in H^{2}\left(\Omega_{j}\right)$ for $j=1,2, p_{\gamma} \in$ $H^{1+\tilde{\alpha}}(\gamma)$, for some $\alpha, \tilde{\alpha} \in\left(\frac{1}{2}, \frac{3}{2}\right)$, and $\lambda=\llbracket \mathbf{K} \nabla p \cdot \mathbf{n}_{\gamma} \rrbracket_{\gamma} \in H^{\frac{1}{2}}(\gamma)$. If $r_{\lambda} \in H^{1}(\Omega)$ is defined as in Lemma 5 with $\zeta=\lambda$, it follows that

$$
\begin{aligned}
& \left\|\left(p-\mathcal{I}_{h}^{\Omega} p, p_{\gamma}-\mathcal{I}_{h}^{\gamma} p_{\gamma} ; \lambda-\left.\left(\pi_{h} r_{\lambda}\right)\right|_{\gamma}\right)\right\|_{1, h} \\
& \quad \leq C_{10}\left(h^{\alpha}\|p\|_{1+\alpha, \Omega}+h_{\gamma}^{\tilde{\alpha}}\left\|p_{\gamma}\right\|_{1+\tilde{\alpha}, \gamma}+h \sum_{j=1,2}\|p\|_{2, \Omega_{j}}\right) .
\end{aligned}
$$

Proof Using the definition of the norm $\mid\|\cdot\|_{0, h}$ we have

$$
\begin{gathered}
\left\|\left(p-\mathcal{I}_{h}^{\Omega} p, p_{\gamma}-\mathcal{I}_{h}^{\gamma} p_{\gamma} ; \lambda-\pi_{h} r_{\lambda}\right)\right\|_{0, h}^{2}=\left\|\nabla\left(p-\mathcal{I}_{h}^{\Omega} p\right)\right\|_{0, \Omega}^{2} \\
\quad+\left\|\nabla_{\tau}\left(p_{\gamma}-\mathcal{I}_{h}^{\gamma} p_{\gamma}\right)\right\|_{0, \gamma}^{2}+\left\|\left.\left(p-\mathcal{I}_{h}^{\Omega} p\right)\right|_{\gamma}\right\|_{\frac{1}{2}, h, \gamma}^{2} \\
+\left\|p_{\gamma}-\mathcal{I}_{h}^{\gamma} p_{\gamma}\right\|_{\frac{1}{2}, h, \gamma}^{2}+\left\|\lambda-\left.\left(\pi_{h} r_{\lambda}\right)\right|_{\gamma}\right\|_{-\frac{1}{2}, h, \gamma}^{2}
\end{gathered}
$$

The first two terms can be controlled using the interpolation estimates (37). For the third term, using (36), and again (37), we obtain

$$
\begin{aligned}
\left\|\left.\left(p_{h}-\mathcal{I}_{h}^{\Omega} p_{h}\right)\right|_{\gamma}\right\|_{\frac{1}{2}, h, \gamma}^{2} & =\sum_{T \in \mathcal{S}_{h}}\left\|\left.\left(p_{h}-\mathcal{I}_{h}^{\Omega} p_{h}\right)\right|_{\gamma}\right\|_{\frac{1}{2}, h, T \cap \gamma}^{2} \\
& \leq \sum_{T \in \mathcal{S}_{h}} C_{t r}\left(h^{-2}\left\|p_{h}-\mathcal{I}_{h}^{\Omega} p_{h}\right\|_{0, T}^{2}+\left\|\nabla\left(p_{h}-\mathcal{I}_{h}^{\Omega} p_{h}\right)\right\|_{0, T}^{2}\right) \\
& \leq \sum_{T \in \mathcal{S}_{h}} 2 C_{t r} C_{\mathcal{I}_{\Omega}}^{2} h^{2 \alpha}|p|_{1+\alpha, T}^{2} \leq 2 C_{t r} C_{\mathcal{I}_{\Omega}}^{2} h^{2 \alpha}|p|_{1+\alpha, \Omega}^{2} .
\end{aligned}
$$

To control the fourth term we need only (37)

$$
\left\|p_{\gamma}-\mathcal{I}_{h}^{\gamma} p_{\gamma}\right\|_{\frac{1}{2}, h_{\gamma}, \gamma} \leq h_{\gamma}^{-\frac{1}{2}} C_{\mathcal{I}_{\gamma}} h_{\gamma}^{1+\tilde{\alpha}}\left|p_{\gamma}\right|_{1+\tilde{\alpha}, \gamma}=C_{\mathcal{I}_{\gamma}} h_{\gamma}^{\frac{1}{2}+\tilde{\alpha}}\left|p_{\gamma}\right|_{1+\tilde{\alpha}, \gamma}
$$


For the fifth term we use Lemma 5 together with the definition of $\lambda$ and (35):

$$
\|\lambda\|_{\frac{1}{2}, \gamma}=\left\|\llbracket \mathbf{K} \nabla p \cdot \mathbf{n}_{\gamma} \rrbracket_{\gamma}\right\|_{\frac{1}{2}, \gamma} \leq \sum_{j=1,2} C_{\mathbf{K}}^{M} \widetilde{C}_{t r}\|\nabla p\|_{1, \Omega_{j}} \leq C_{\mathbf{K}}^{M} \widetilde{C}_{t r} \sum_{j=1,2}\|p\|_{2, \Omega_{j}}
$$

The stabilizing term $\mathcal{J}\left(\lambda_{h}, \lambda_{h}\right)^{\frac{1}{2}}$ is controlled similarly by the second estimate of Lemma 5. Thus the proof is completed.

Theorem 2 Again, let $P=\left(p, p_{\gamma} ; \lambda\right) \in \boldsymbol{V} \times \Lambda$ be the solution of (3) and let $P_{h}=\left(p_{h}, p_{\gamma, h} ; \lambda_{h}\right) \in \boldsymbol{V}_{h} \times \Lambda_{h}$ be the solution of (8), and suppose the same regularity as in Lemma 6 . Then there exists a constant $C$ such that

$$
\left\|P-P_{h}\right\|_{0, h}+\mathcal{J}\left(\lambda_{h}, \lambda_{h}\right)^{\frac{1}{2}} \leq C\left(h^{\alpha}\|p\|_{1+\alpha, \Omega}+h_{\gamma}^{\tilde{\alpha}}\left\|p_{\gamma}\right\|_{1+\tilde{\alpha}, \gamma}+h \sum_{j=1,2}\|p\|_{2, \Omega_{j}}\right) .
$$

Proof In Proposition 3 choose $q_{h}=\mathcal{I}_{h}^{\Omega} p, q_{\gamma, h}=\mathcal{I}_{h}^{\gamma} p_{\gamma}$ and $\mu_{h}=\pi_{h} r_{\lambda}$ where $r_{\lambda}$ is defined as in Lemma 5 with $\zeta=\lambda$. Then the estimate follows directly from Lemma 6.

Remark 1 Generally $p$ is expected to belong to $H^{3 / 2-\varepsilon}(\Omega)$ for $\varepsilon>0$, and it is reasonable to assume that $p_{\gamma}$ belongs to $H^{2}(\gamma)$ (when $f_{\gamma}$ is regular for instance) and that $\left.p\right|_{\Omega_{j}} \in H^{2}\left(\Omega_{j}\right)$. Thus $\left.\left(\left.\mathbf{K} \nabla p\right|_{\Omega_{j}} \cdot \mathbf{n}_{\gamma}\right)\right|_{\gamma}$ belongs to $H^{\frac{1}{2}}(\gamma)$, and the jump $\lambda$ is indeed in $H^{\frac{1}{2}}(\gamma)$. Thus Theorem 2 provides generally a suboptimal $\mathcal{O}\left(h^{\frac{1}{2}-\varepsilon}\right)$ convergence. However a better rate of convergence of $\mathcal{O}(h)$ is frequently observed in practice.

\section{Numerical results}

This section is devoted to numerical experiments studying the proposed discretization (8). In particular we address accuracy, convergence and conditioning for various choices of the penalization parameter. A direct solver is used to solve the linear system. While in the theoretical considerations we have discussed only the case of a single fracture, we have looked at some numerical examples in a few more complex cases. These were treated in the simplest fashion: we assumed continuity of pressure and mass conservation at the intersection of fractures and no flow into or out of a fracture through a tip that is neither a tip of another fracture nor on the boundary of the domain. The simplest way to treat the multipliers is to assume that fractures don't cross but simply meet at their tips: 2 fractures crossing each other can be considered as 4 fractures with one tip in common. (Similarly a sharp angle at a point in a fracture would cause it to be considered as 2 fractures having this point as a tip.) In this way jumps in the multipliers at "crossing points" (or at "irregular points") are not penalized. Of course for a given data set of fractures it may be simpler to simply introduce extra multipliers at crossing points (and at irregular points). Moreover the winding number algorithm is used if the fracture lies along or contains vertices of the matrix mesh. 


\subsection{Case 1: a vertical fracture}

The first setup is a two-dimensional, square domain $\Omega:=[0,1]^{2}$ with homogeneous Neumann conditions on the horizontal boundaries and nonhomogeneous Dirichlet conditions on the vertical boundaries $(p=1$ on the left and $p=4$ on the right). A vertical fracture $\gamma$ with $\mathbf{K}_{\gamma}=10, \mathbf{K}=\mathbf{I}$, is located in the middle of the matrix domain $\Omega$ with Dirichlet boundary values $p_{\gamma}=1$ on the lower tip and $p_{\gamma}=4$ on the upper tip. The test case and the pressure distribution are shown in Fig. 2. The simulations are performed for various mesh sizes $h, h_{\gamma}$
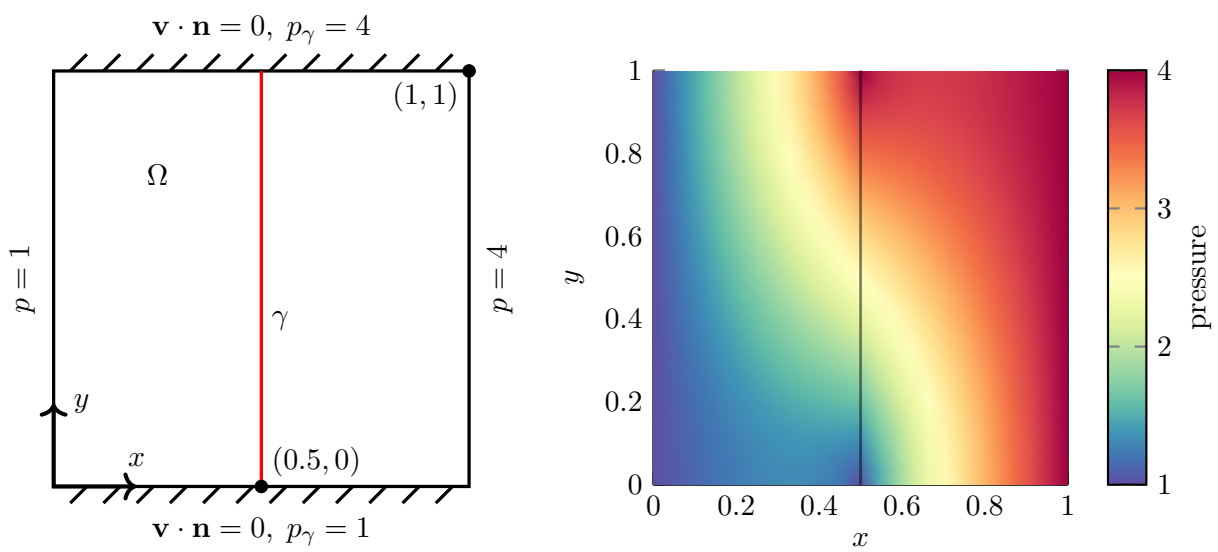

Fig. 2 Case 1 (a vertical fracture): General setting (left) and distribution of the pressures (right) of a simulation with $h=1 / 24, h_{\gamma}=1 / 48, \xi=1$.

and $h_{\lambda}$. However, due to the high permeability in the vertical fracture, we have taken $h_{\gamma}=h / 2$ in order to obtain high accuracy at the fracture interface. In the simulations the fracture is either located on the edges of the rectangular matrix grid, referred to as conforming, or in the center of the rectangular elements, referred to as nonconforming.

On this basis we study the performance of the method. As the analytical solution is not known, a fine simulation of the stabilized Lagrange multiplier method with mesh size $h=1 / 384$ is used as a reference solution. The influence of the penalty value $\xi$ on accuracy, convergence and conditioning is analyzed in the conforming as well as the nonconformig case.

Fig. 3 presents the numerical convergence analysis of the primary variables for $\xi=0.5$ in both the cases of conforming and nonconforming meshes. As predicted by the theory and independently of the mesh configuration, the errors for $p$ and $p_{\gamma}$ converge linearly in the $H^{1}$ norm and the Lagrange multiplier converges linearly in the norm $\|\cdot\|_{-\frac{1}{2}, \gamma, h}$. Depending on the mesh configuration the $L^{2}$ errors of the pressures converge with rates up to quadratic order: in the nonconforming case the matrix pressure converges linearly in the $L^{2}$ norm.

Figs. 4 and 5 display the approximation errors for matrix pressure, fracture pressure and the Lagrange multiplier for different penalty parameters $\xi$ and dif- 


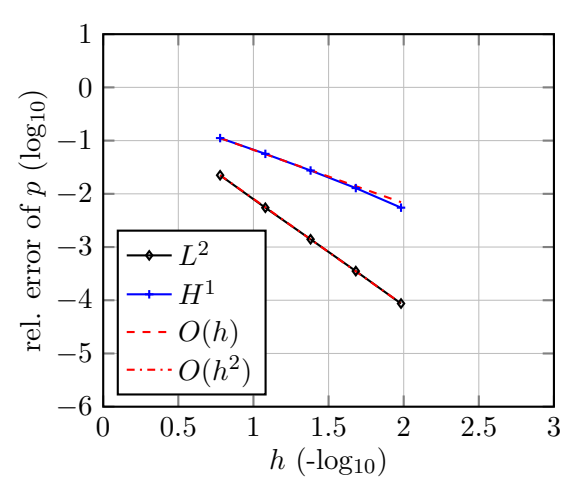

(a) Error for $p$ (conforming)

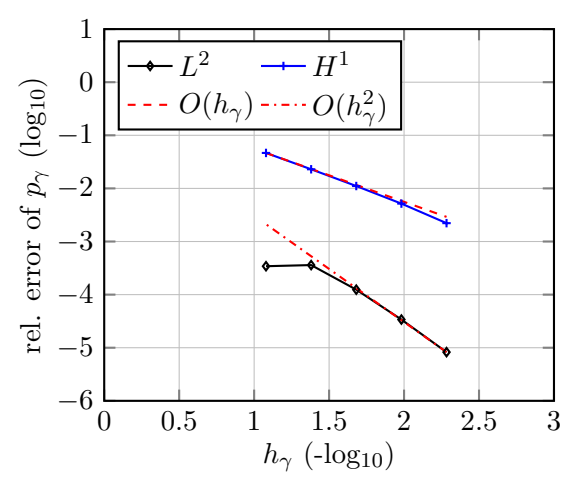

(c) Error for $p_{\gamma}$ (conforming)

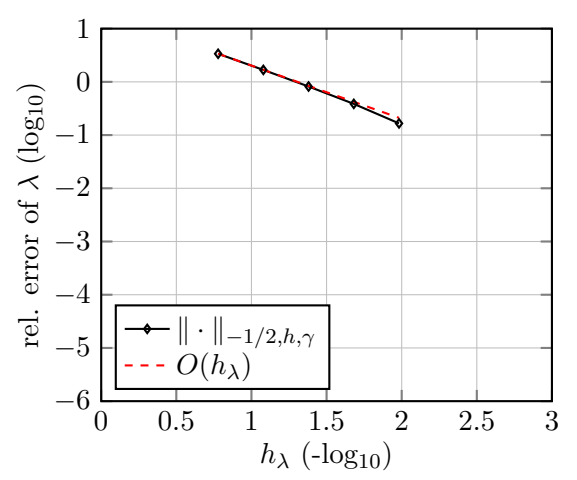

(e) Error for $\lambda$ (conforming)

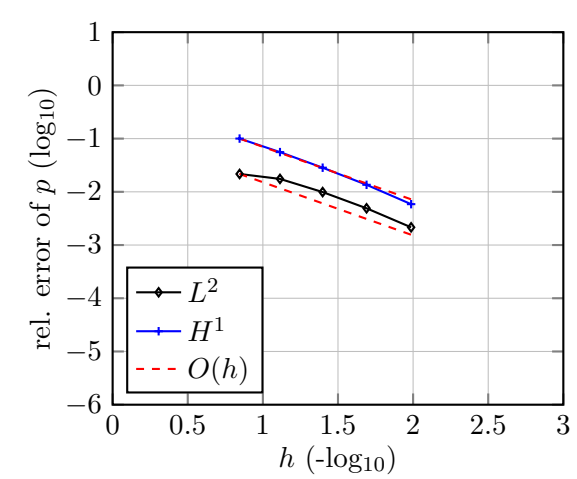

(b) Error for $p$ (nonconforming)

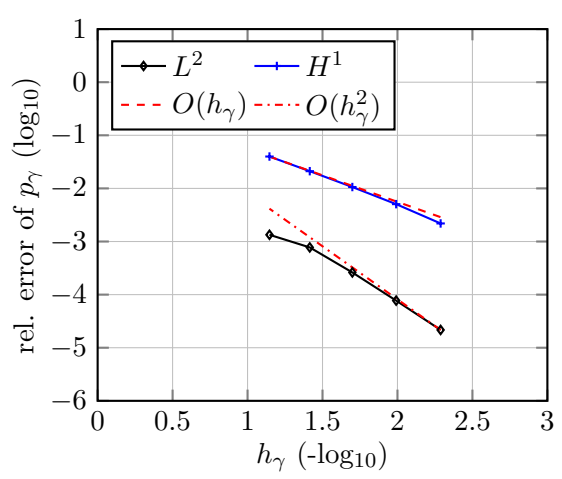

(d) Error for $p_{\gamma}$ (nonconforming)

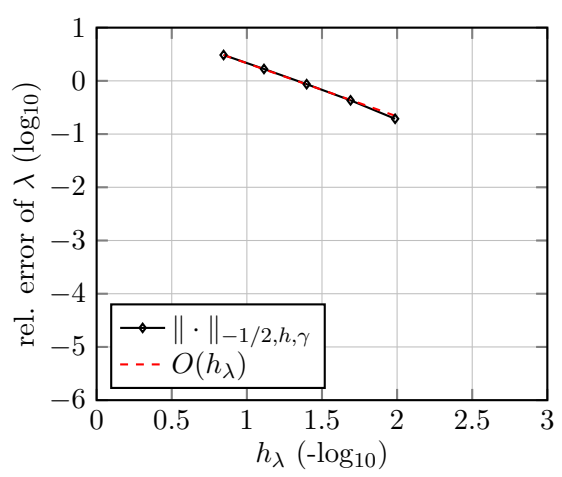

(f) Error for $\lambda$ (nonconforming)

Fig. 3 Case 1 (a vertical fracture): Convergence of matrix pressure $p$, fracture pressure $p_{\gamma}$ and Lagrange multiplier $\lambda$ for $\xi=0.5$ in the conforming case (left) and the nonconforming case (right) depending on the mesh size. 


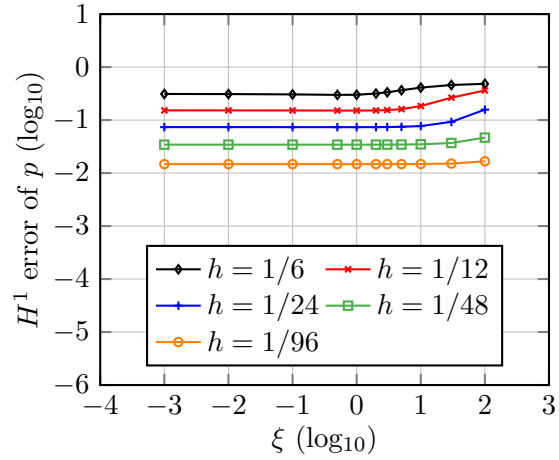

(a) $H^{1}$ Error for $p$ (conforming)

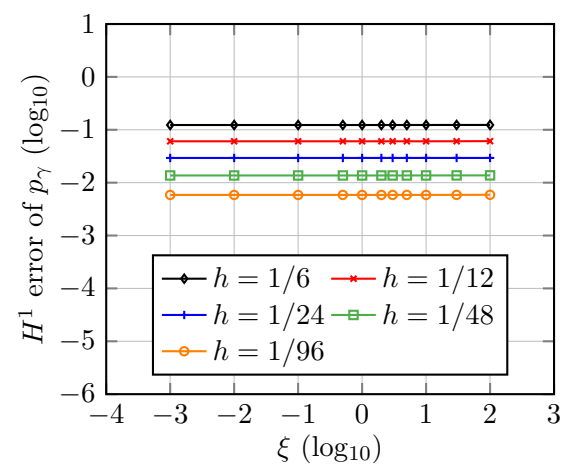

(c) $H^{1}$ Error for $p_{\gamma}$ (conforming)

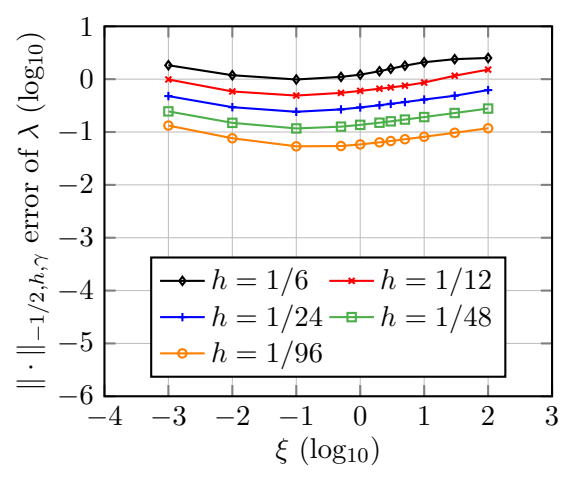

(e) Error for $\lambda$ (conforming)

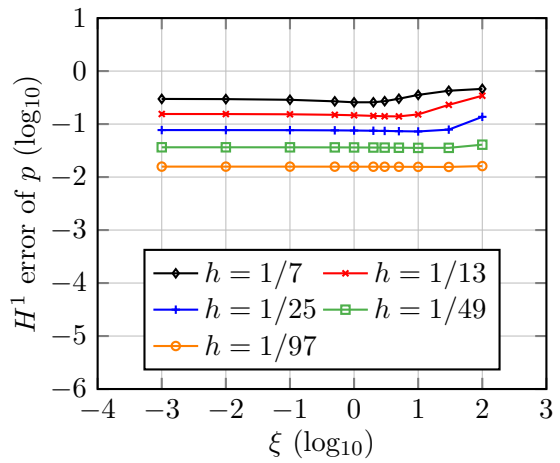

(b) $H^{1}$ Error for $p$ (nonconforming)

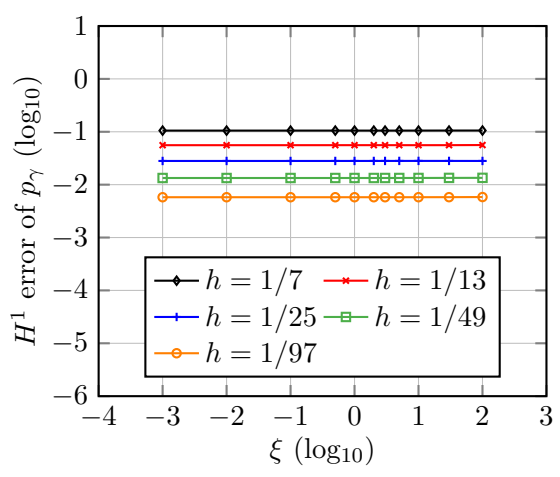

(d) $H^{1}$ Error for $p_{\gamma}$ (nonconforming)

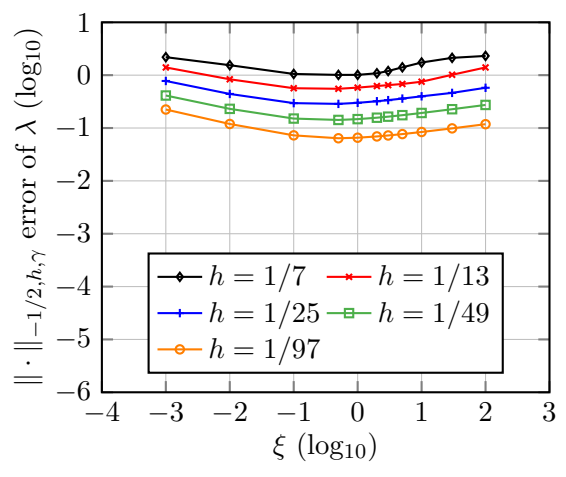

(f) Error for $\lambda$ (nonconforming)

Fig. 4 Case 1 (a vertical fracture): Influence of the penalty value $\xi$ on the $H^{1}$ error for the matrix pressure $p$, the fracture pressure $p_{\gamma}$ and on the $\|\cdot\|_{-\frac{1}{2}, h, \gamma}$ error for the Lagrange multiplier $\lambda$ in the conforming case (left) and in the nonconforming case (right) depending on the mesh refinement index $h$. 


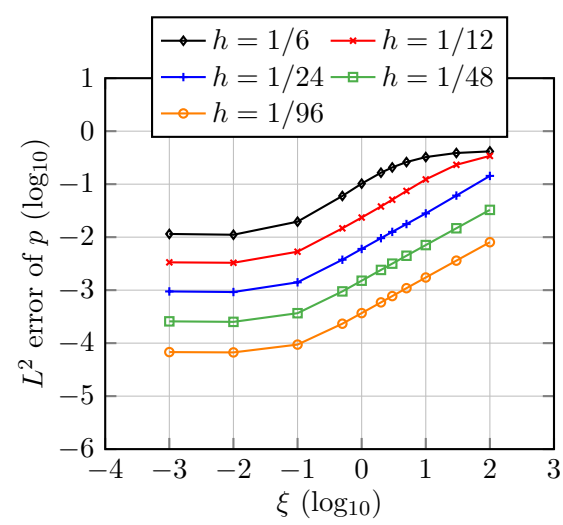

(a) $L^{2}$ Error for $p$ (conforming)

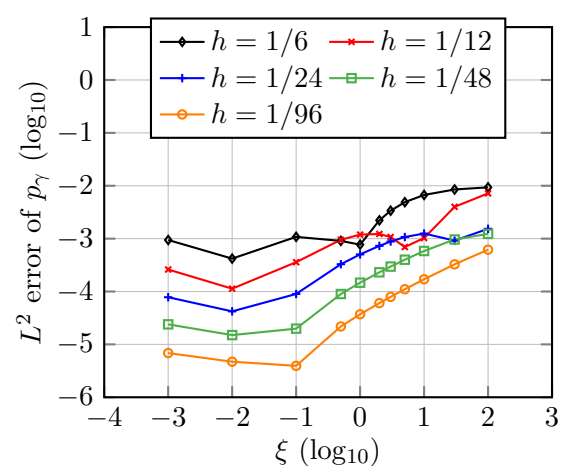

(c) $L^{2}$ Error for $p_{\gamma}$ (conforming)

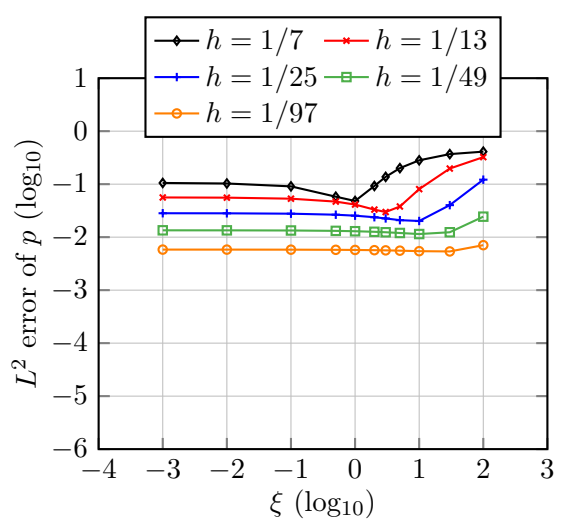

(b) $L^{2}$ Error for $p$ (nonconforming)

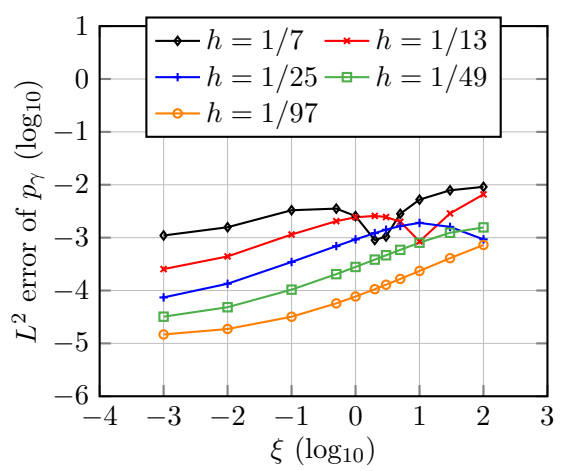

(d) $L^{2}$ Error for $p_{\gamma}$ (nonconforming)

Fig. 5 Case 1 (a vertical fracture): Influence of the penalty value $\xi$ on the $L^{2}$ approximation error for the matrix pressure $p$ and the fracture pressure $p_{\gamma}$ in the conforming case (left) and in the nonconforming case (right) depending on the mesh refinement index $h$.

ferent mesh sizes in the conforming and nonconforming cases. The plots indicate that the errors get smaller when the mesh is refined. The penalty parameter $\xi$ has little influence on the $H^{1}$ errors for $p$ and $p_{\gamma}$ and seems to give slightly improved results for $\lambda$ when $\xi \in[0.1,1]$, see Fig. 4. The influence of $\xi$ is more important for $p$ and $p_{\gamma}$ in the $L^{2}$ error, see Fig. 5. In the most interesting case (nonconforming), small to moderate penalty values $(\xi \leq 1)$ seem optimal for the accuracy, since the penalty term aligns the multipliers at the expense of the coupling accuracy between matrix and fracture. In each plot, looking at the different curves for fixed $\xi$, one can determine the convergence rate, that is shown in Fig. 3 for $\xi=0.5$. Globally, the smaller the mesh size $h$, the lesser the influence of the penalty parameter $\xi$. Furthermore an increase of the penalty parameter $\xi$ generally leads to higher errors, which is a logical consequence of the construction of $\mathcal{J}$. We also observe in Fig. 5 that the nonconforming configu- 


\begin{tabular}{lllllll}
\hline Distance & $\xi=0$ & $\xi=0.001$ & $\xi=0.01$ & $\xi=0.1$ & $\xi=1$ & $\xi=10$ \\
\hline$h / 2$ & $4.06 \mathrm{e}+8$ & $9.93 \mathrm{e}+7$ & $1.19 \mathrm{e}+7$ & $2.35 \mathrm{e}+6$ & $9.19 \mathrm{e}+5$ & $5.18 \mathrm{e}+5$ \\
$h / 4$ & $3.31 \mathrm{e}+8$ & $9.50 \mathrm{e}+7$ & $1.18 \mathrm{e}+7$ & $2.25 \mathrm{e}+6$ & $8.88 \mathrm{e}+5$ & $5.08 \mathrm{e}+5$ \\
$h / 8$ & $2.69 \mathrm{e}+8$ & $8.97 \mathrm{e}+7$ & $1.18 \mathrm{e}+7$ & $2.14 \mathrm{e}+6$ & $8.53 \mathrm{e}+5$ & $4.96 \mathrm{e}+5$ \\
0 & $2.14 \mathrm{e}+8$ & $8.31 \mathrm{e}+7$ & $1.18 \mathrm{e}+7$ & $2.01 \mathrm{e}+6$ & $8.08 \mathrm{e}+5$ & $4.81 \mathrm{e}+5$ \\
\hline
\end{tabular}

Table 1 Case 1 (a vertical fracture): Condition numbers as a function of $\xi$ and the distance between the matrix mesh edges and the fracture line for $h=1 / 18, h_{\gamma}=1 / 36$.

ration of the mesh yields larger $L^{2}$ errors, errors that have a weaker dependence on $\xi$.

In addition to the nonconforming case in which the fracture cuts through the middle of a vertical strip of matrix mesh elements, we wish to study what happens as the matrix mesh edges approach the fracture line. Table 1 shows the condition numbers of the system matrix depending on the penalty parameter $\xi$ and the distance between the matrix mesh edge and the fracture line for $h=1 / 18, h_{\gamma}=1 / 36$. The table indicates that the location of the fracture within the matrix grid does not influence significantly the conditioning of the system. However the condition number decreases with increasing values of the penalty parameter. This verifies the efficiency of the stabilizing term $\mathcal{J}$.

We conclude that the method (8) behaves as predicted by the theoretical results. The penalty parameter $\xi$ should be chosen depending on accuracy and conditioning of the system matrix. The stabilizing term $\mathcal{J}$ penalizes the jumps of the Lagrange multiplier. The higher the penalty parameter $\xi$ the more the discrete multipliers tend to a constant value which affects the accuracy. However the results show clear convergence also for larger $\xi$. On the other hand high penalty parameters improve the conditioning.

Remark 2 We have not included comparisons with the non-penalized Lagrange multiplier method of Köppel et al (2018) for lack of space. For this comparison see Köppel (2018).

\subsection{Case 2: a fracture network}

In this section a more complex test case with a regular fracture network, $c f$. (Geiger et al, 2013; Flemisch et al, 2018), is considered. The setup and the pressure distribution for a simulation with the stabilized method with nonconforming configuration and $h=1 / 33, h_{\gamma}=1 / 32, \xi=1$, is illustrated in Fig. 6. All fractures of the test case are conductive and have a uniform permeability of $\mathbf{K}_{\gamma}=1$. The unit square matrix domain is characterized by a permeability of $\mathbf{K}=\mathbf{I}$. Throughout this section the mesh size of the fracture will be in the same range as the mesh size of the matrix, i.e. $h_{\gamma} \approx h$.

In Table 2 we compare the method (8) with several other available methods given in the benchmark study Flemisch et al (2018) for single-phase flow in fractured porous media. The reference solution is computed with a mimetic finite difference method (Brezzi et al, 2005) with a two-dimensional grid in the fracture as well as in the matrix domain. The table shows that the stabilized Lagrange multiplier method performs well. Intermediate values of the penalty 

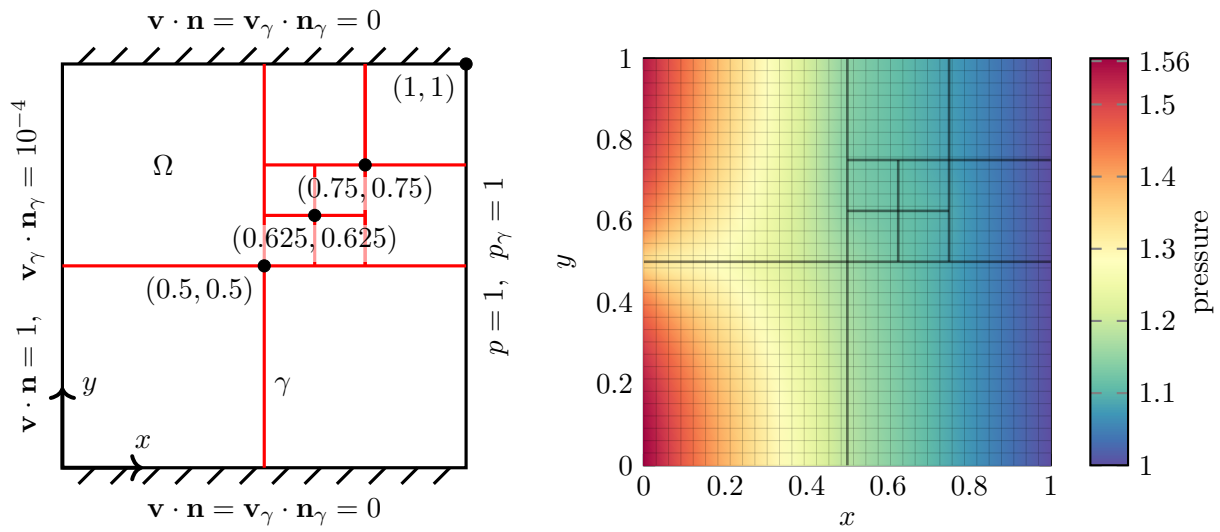

Fig. 6 Case 2 (a fracture network): General setup (left) and pressure distribution (right) of a nonconforming simulation with $h=1 / 33, h_{\gamma}=1 / 32, \xi=1$.

\begin{tabular}{lllllll}
\hline method & d.o.f. & \#-matr & \#-frac & $\operatorname{err}_{m}$ & $\operatorname{err}_{\gamma}$ & cond \\
\hline SLM-FEM: $\xi=0$ & 1374 & 1089 quads & 112 & $1.0 \mathrm{e}-2$ & $6.5 \mathrm{e}-3$ & $6.9 \mathrm{e}+8$ \\
SLM-FEM: $\xi=0.1$ & 1374 & 1089 quads & 112 & $1.0 \mathrm{e}-2$ & $6.5 \mathrm{e}-3$ & $2.0 \mathrm{e}+6$ \\
SLM-FEM: $\xi=1$ & 1374 & 1089 quads & 112 & $9.9 \mathrm{e}-3$ & $6.4 \mathrm{e}-3$ & $7.7 \mathrm{e}+5$ \\
SLM-FEM: & 1374 & 1089 quads & 112 & $6.4 \mathrm{e}-2$ & $2.9 \mathrm{e}-2$ & $1.3 \mathrm{e}+5$ \\
$\xi=1000$ & & & & & & \\
\hline Box & 577 & 1078 trias & 74 & $1.1 \mathrm{e}-2$ & $1.9 \mathrm{e}-4$ & $2.2 \mathrm{e}+3$ \\
MPFA & 1439 & 1348 trias & 91 & $1.1 \mathrm{e}-2$ & $4.5 \mathrm{e}-3$ & $5.8 \mathrm{e}+4$ \\
EDFM & 1501 & 1369 quads & 132 & $6.5 \mathrm{e}-3$ & $4.0 \mathrm{e}-3$ & $5.6 \mathrm{e}+4$ \\
Flux-Mortar & 3366 & 1280 trias & 75 & $1.0 \mathrm{e}-2$ & $6.9 \mathrm{e}-3$ & $2.4 \mathrm{e}+6$ \\
P-XFEM & 1650 & 961 quads & 164 & $9.3 \mathrm{e}-3$ & $7.3 \mathrm{e}-3$ & $9.3 \mathrm{e}+9$ \\
D-XFEM & 4474 & 1250 trias & 126 & $9.6 \mathrm{e}-3$ & $8.9 \mathrm{e}-3$ & $1.2 \mathrm{e}+6$ \\
\hline
\end{tabular}

Table 2 Case 2 (a fracture network): Performance of the stabilized Lagrange multiplier method (SLM-FEM) compared to other DFM methods, cf. Flemisch et al (2018), based on the number of degrees of freedom (d.o.f.), the number of matrix elements (\#-matr), the number of fracture elements (\#-frac), the matrix error $\left(\operatorname{err}_{m}\right)$, the fracture error $\left(\operatorname{err}_{\gamma}\right)$ and the condition number (cond).

parameter, i.e. $\xi \in[0.1,1]$, yield a good balance between accuracy and conditioning. Note however that this range may change for more realistic permeabilities, see Section 4.3.

To study the numerical convergence of the stabilized method the configuration is refined three times by a factor of two $(h \in\{1 / 33,1 / 65,1 / 129,1 / 257\})$ similar to Flemisch et al (2018). The resulting convergence study of matrix and fracture pressure using the stabilized discretization with $\xi=1$ is illustrated in Fig. 7. The figure shows that the stabilized method converges linearly in the matrix and in the fracture as the other methods of the benchmark study. 

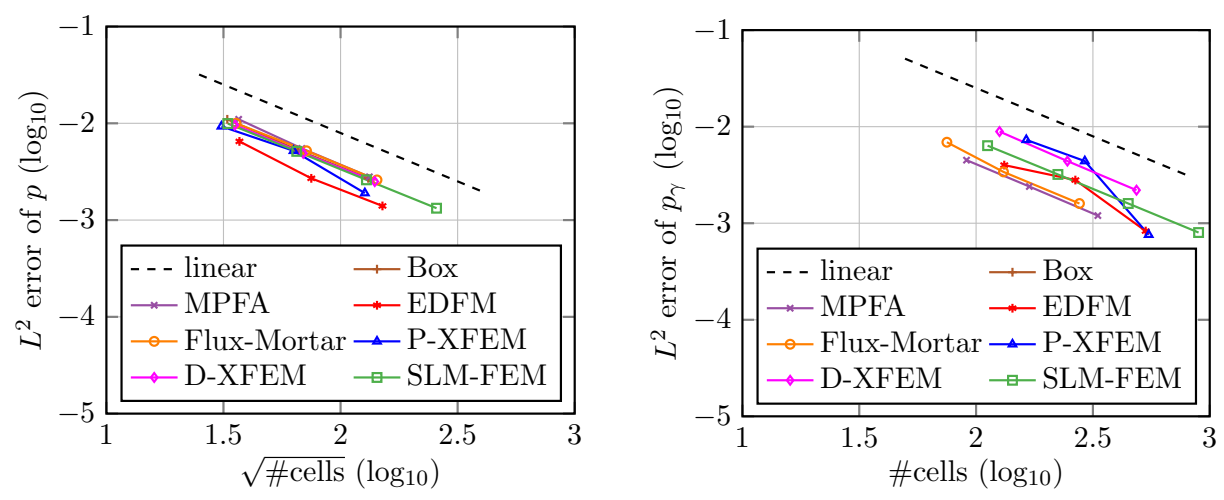

Fig. 7 Case 2 (a fracture network): Convergence of matrix pressure $p$ (left) and fracture pressure $p_{\gamma}$ (right) of the stabilized Lagr. multiplier method (SLM-FEM) for $\xi=1$ depending on the number of elements (\#cells) compared to other methods, $c f$. Flemisch et al (2018).

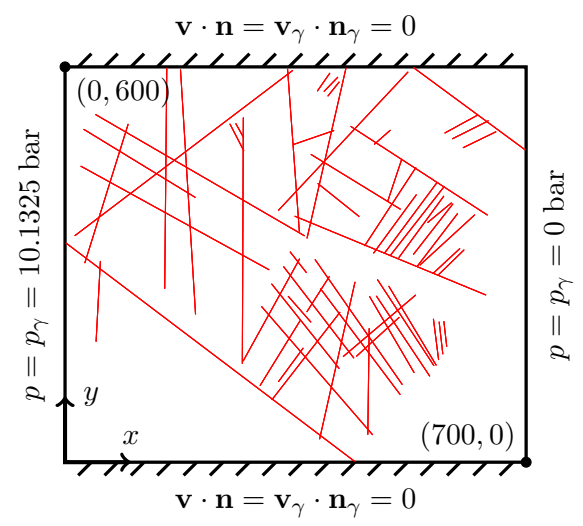

Fig. 8 Case 3 (a realistic case): Setup. The fractures are depicted as red lines.

\subsection{Case 3: a realistic case}

The last numerical experiment represents a real set of fractures from an interpreted outcrop in the Sotra island near Bergen in Norway of the benchmark study Flemisch et al (2018). The domain is rectangular with uniform permeability $\mathbf{K}=10^{-14} \mathrm{~m}^{2}$. It contains 64 fractures grouped in several networks with $\mathbf{K}_{\gamma}=10^{-10} \mathrm{~m}^{3}$, see Fig. 8 . The size of the domain is $700 \mathrm{~m} \times 600 \mathrm{~m}$ with homogeneous Neumann boundary conditions on top and bottom, a pressure of 10.1325 bar on the left and a pressure of 0 bar on the right.

The pressure distributions for simulations with $h=10 \mathrm{~m}$ and $\xi=1$ and 100 are displayed in Fig. 9. A small undershoot can be observed in the vicinity of the upper right fracture connecting the top with the right boundary. In this region the pressures should be similar to the boundary condition on the right. Because 

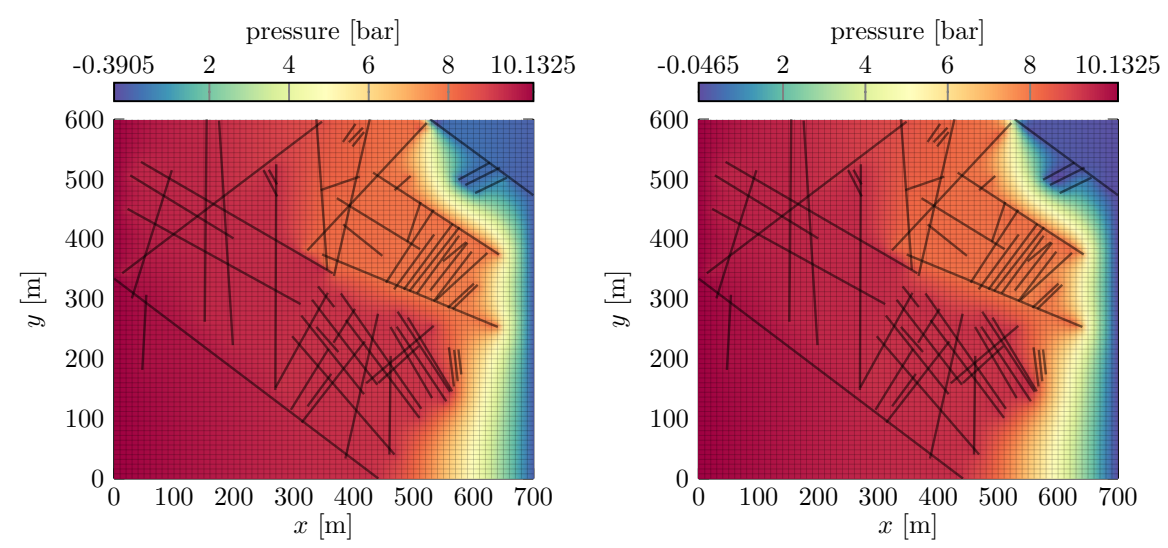

Fig. 9 Case 3 (a realistic case): Pressure distribution. Simulation with $h=10 \mathrm{~m}$ and for $\xi=1$ (left) and $\xi=100$ (right). The lowest value is slightly different for the two figures.

of the piecewise linear basis functions and the high permeability contrast, the weak constraint of the equality of the pressures on the fracture interface leads to unphysical matrix pressures near the fracture. However the undershoot is smaller for the larger choice of $\xi$. Fig. 10 shows the distributions of the pressures at $y=500 \mathrm{~m}$ and $x=625 \mathrm{~m}$ of the stabilized Lagrange multiplier method compared to other methods of the benchmark study. The stabilized Lagrange multiplier method is in very good agreement with the pressure distributions of the other methods. A convergence analysis was not performed in this test case, as was noted in Flemisch et al (2018), since it is a really difficult task to establish a full-dimensional reference solution with the mimetic finite difference method. The comparison in terms of the conditioning and degrees of freedom is given in Table 3 and shows good performances with moderate to large values of $\xi$.

Remark 3 In order to improve the conditioning in this realistic test case where the permeabilities are very small, a scaling was performed prior to the computations: the duality pairings $\langle\cdot, \cdot\rangle_{\gamma}$ of $\mathcal{A}$ defined in (2) and the stabilization term $\mathcal{J}$ defined in (6) were multiplied by the scalar $k=\mathbf{K}$. Thus the actual Lagrange multiplier unknown was $\tilde{\lambda}=k \lambda$, and the stabilization term $\mathcal{J}$ scales correctly with $\mathbf{K}$. It was then possible to divide the discrete system (8) by $\mathbf{K}_{\gamma}$, and obtain a system that is easier to solve. In the case when $\mathbf{K}$ is a tensor or varying in space, the same idea should be considered with an average value of its norm.

\section{Conclusion}

In this paper we presented a stabilized finite element discretization of a Lagrange multiplier model for single-phase Darcy flow in fractured porous media, $c f$. Köppel et al (2018), where the multiplier represents a local exchange of the fluid between fracture and matrix domain allowing for the use of a mesh in the 

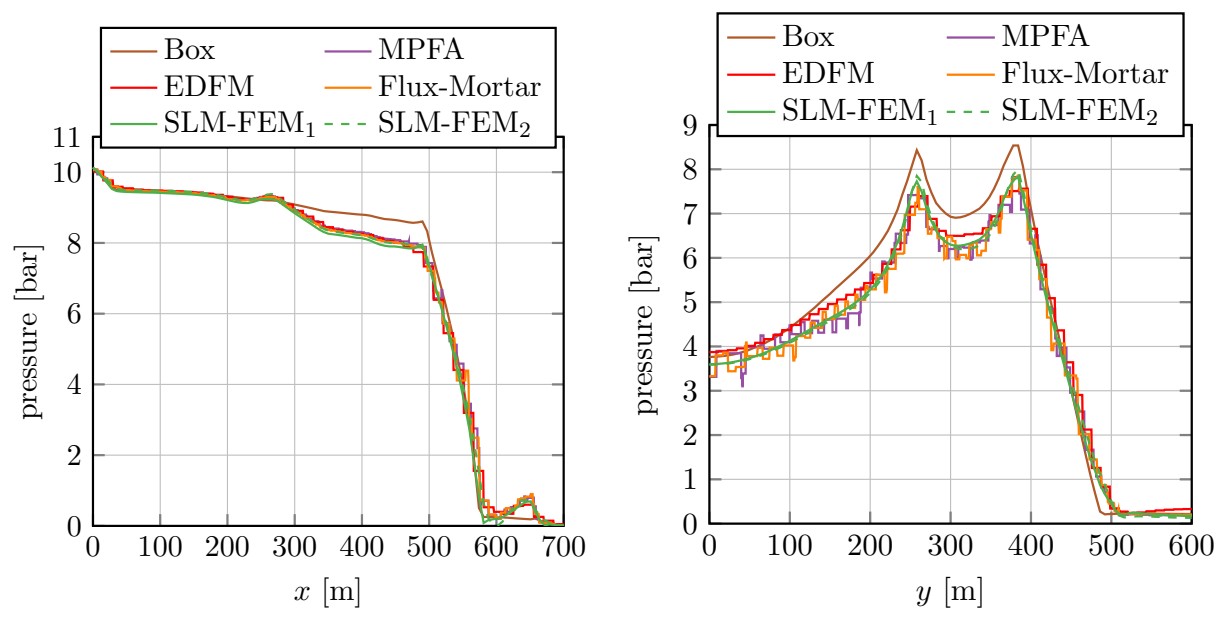

Fig. 10 Case 3 (a realistic case): Pressure distribution along the line $y=500 \mathrm{~m}$ (left) and along the line $x=625 \mathrm{~m}$ (right) of the stabilized Lagrange multiplier method (with $h=10 \mathrm{~m}$ ) in comparison with other methods, $c f$. Flemisch et al (2018). SLM-FEM 1 : $\xi=1$. SLM-FEM $2: \xi=100$.

\begin{tabular}{lllll}
\hline method & d.o.f. & \#-matr & \#-frac & cond \\
\hline SLM-FEM: $\xi=0$ & 8081 & 5250 quads & 1372 & $4.8 \mathrm{e}+9$ \\
SLM-FEM: $\xi=1 \mathrm{e}-4$ & 8081 & 5250 quads & 1372 & $3.5 \mathrm{e}+8$ \\
SLM-FEM: $\xi=1$ & 8081 & 5250 quads & 1372 & $6.1 \mathrm{e}+6$ \\
SLM-FEM: $\xi=100$ & 8081 & 5250 quads & 1372 & $6.2 \mathrm{e}+6$ \\
\hline Box & 5563 & 10807 trias & 1386 & $9.3 \mathrm{e}+5$ \\
MPFA & 8588 & 7614 trias & 867 & $4.9 \mathrm{e}+6$ \\
EDFM & 3599 & 2491 quads & 1108 & $4.7 \mathrm{e}+6$ \\
Flux-Mortar & 25258 & 8319 trias & 1317 & $2.2 \mathrm{e}+17$ \\
\hline
\end{tabular}

Table 3 Benchmark 3 (a realistic case): Performance of the stabilized Lagrange multiplier method (SLM-FEM) compared to other DFM methods based on the number of degrees of freedom (d.o.f.), the number of matrix elements (\#-matr), the number of fracture elements (\#-frac) and the condition number (cond), cf. Flemisch et al (2018).

matrix that is not aligned with the fracture. The piecewise constant Lagrange multipliers of the stabilized discretization are defined on the intersections of the matrix elements with the fracture and, hence, are embedded on the fracture interface. In contrast to the method proposed in Köppel et al (2018), a weakly consistent stability term penalizes the jumps of the consecutive multipliers to stabilize the discrete saddle point system. We proved stability and convergence of the discrete formulation following the ideas of Burman and Hansbo (2010a). The numerical experiments are consistent with the theoretical results. They confirmed that with increasing values of the penalty parameter $\xi$ the conditioning of the discrete system can be improved. On the other hand high penalty values deteriorate the accuracy of the approximation. Hence we recommend the use of intermediate penalty values to obtain optimal results. The particular choice of $\xi$ depends on the test case considered. Despite the affected accuracy the results 
still show clear convergence even for large penalty parameters. In the numerical examples, when the coupling term $\lambda$ is regular enough the errors of the matrix and fracture pressure converge linearly in the $H^{1}$ norm confirming the theoretical results. The Lagrange multiplier is characterized by linear rates of convergence in the discrete norm $\|\cdot\|_{-\frac{1}{2}, h_{\lambda}, \gamma}$. These convergence rates are obtained independently of the possibly very irregular induced mesh for the Lagrange multiplier. The comparison with the benchmark results in Flemisch et al (2018), leads to the conclusion that the penalized discretization is a good alternative to other models for the simulation of flow in fractured porous media.

Acknowledgements The authors would like to thank the German Research Foundation (DFG) for financial support of the project within the Cluster of Excellence in Simulation Technology (EXC 310/2) at the University of Stuttgart, and the two referees whose comments helped improving this paper. .

\section{References}

Ahmed E, Jaffré J, Roberts JE (2017) A reduced fracture model for two-phase flow with different rock types. Math Comput Simulation 137:49-70

Ainsworth M (2007) A Posteriori Error Estimation for Discontinuous Galerkin Finite Element Approximation. SIAM J Numer Anal 45(4):1777-1798

Alboin C, Jaffré J, Roberts JE, Serres C (2002) Modeling fractures as interfaces for flow and transport in porous media. In: Fluid flow and transport in porous media: mathematical and numerical treatment, Contemp. Math., vol 295, Amer. Math. Soc., Providence, RI, pp 13-24

Angot P, Boyer F, Hubert F (2009) Asymptotic and numerical modelling of flows in fractured porous media. ESAIM: M2AN 43(2):239-275

Antonietti PF, Facciolà C, Russo A, Verani M (2016a) Discontinuous Galerkin approximation of flows in fractured porous media on polytopic grids. Tech. Rep. 22/2016, Politecnico di Milano

Antonietti PF, Formaggia L, Scotti A, Verani M, Verzott N (2016b) Mimetic finite difference approximation of flows in fractured porous media. ESAIM: M2AN 50(3):809-832

Baca RG, Arnett RC, Langford DW (1984) Modelling fluid flow in fracturedporous rock masses by finite-element techniques. Int J Numer Methods Fluids 4:337-348

Berrone S, Pieraccini S, Scialò S (2014) An optimization approach for large scale simulations of discrete fracture network flows. J Comput Phys 256:838-853

Boon W, Nordbotten J, Yotov I (2018) Robust Discretization of Flow in Fractured Porous Media. SIAM J Numer Anal 56(4):2203-2233

Brenner K, Groza M, Guichard C, Masson R (2015) Vertex approximate gradient scheme for hybrid dimensional two-phase Darcy flows in fractured porous media. ESAIM Math Model Numer Anal 49(2):303-330

Brezzi F, Lipnikov K, Simoncini V (2005) A family of mimetic finite difference methods on polygonal and polyhedral meshes. Math Models Methods Appl Sci 15(10):1533-1551 
Burman E, Hansbo P (2010a) Fictitious domain finite element methods using cut elements: I. A stabilized Lagrange multiplier method. Comput Methods Appl Mech Engrg 199(41-44):2680-2686

Burman E, Hansbo P (2010b) Interior-penalty-stabilized Lagrange multiplier methods for the finite-element solution of elliptic interface problems. IMA J Numer Anal 30:870-885

Chave FA, Di Pietro DA, Formaggia L (2018) A Hybrid High-Order method for passive transport in fractured porous media, URL https://hal . archives-ouvertes.fr/hal-01784181

Costabel M (1988) Boundary Integral Operators on Lipschitz Domains: Elementary Results. SIAM J Math Anal 19(3):613-626

Ding Z (1996) A Proof of the Trace Theorem of Sobolev Spaces on Lipschitz Domains. Proc Am Math Soc 124(2):591-600

Ern A, Guermond JL (2004) Theory and practice of finite elements, Applied Mathematical Sciences, vol 159. Springer-Verlag, New York

Faille I, Fumagalli A, Jaffré J, Roberts JE (2016) Model reduction and discretization using hybrid finite volumes for flow in porous media containing faults. Comput Geosci 20(2):317-339

Flemisch B, Berre I, Boon W, Fumagalli A, Schwenck N, Scotti A, Stefansson I, Tatomir A (2018) Benchmarks for single-phase flow in fractured porous media. Adv Water Resour 111:239-258

Formaggia L, Scotti A, Sottocasa F (2018) Analysis of a mimetic finite difference approximation of flows in fractured porous media. ESAIM: M2AN 52(2):595630

Frih N, Martin V, Roberts JE, Saâda A (2012) Modeling fractures as interfaces with nonmatching grids. Comput Geosci 16(4):1043-1060

Fumagalli A, Scotti A (2013) A numerical method for two-phase flow in fractured porous media with non-matching grids. Adv Water Resour 62, Part C:454464, computational Methods in Geologic CO2 Sequestration

Fumagalli A, Pasquale L, Zonca S, Micheletti S (2016) An upscaling procedure for fractured reservoirs with embedded grids. Water Resour Res 52(8)

Galvis J, Sarkis M (2007) Non-matching mortar discretization analysis for the coupling Stokes-Darcy equations. Electronic Transactions on Numerical Analysis $26: 350-384$

Geiger S, Dentz M, Neuweiler I (2013) A novel multi-rate dual-porosity model for improved simulation of fractured and multi-porosity reservoirs. Soc Petrol Eng J 18(4):670-684

Girault V, Glowinski R (1995) Error analysis of a fictitious domain method applied to a Dirichlet problem. Jpn J Ind Appl Math 12(3):487

Hoteit H, Firoozabadi A (2008) An efficient numerical model for incompressible two-phase flow in fractured media. Adv Water Resour 31:891-905

Karimi-Fard M, Durlofsky LJ, Aziz K (2004) An efficient discrete-fracture model applicable for general-purpose reservoir simulators. Soc Petrol Eng J 9(2):227236

Knabner P, Roberts JE (2014) Mathematical analysis of a discrete fracture model coupling Darcy flow in the matrix with Darcy-Forchheimer flow in the fracture. ESAIM: Math Model Numer Anal 48(5):1451-1472 
Köppel M (2018) Flow in Heterogeneous Porous Media: Fractures and Uncertainty Quantification. PhD thesis, University of Stuttgart, Germany

Köppel M, Martin V, Jaffré J, Roberts JE (2018) A Lagrange multiplier method for a discrete fracture model for flow in porous media. accepted URL https : //hal.archives-ouvertes.fr/hal-01700663

Lesinigo M, D'Angelo C, Quarteroni A (2011) A multiscale Darcy-Brinkman model for fluid flow in fractured porous media. Numer Math 117(4):717-752

Martin V, Jaffré J, Roberts JE (2005) Modeling Fractures and Barriers as Interfaces for Flow in Porous Media. SIAM J Sci Comput 26(5):1667-1691

Massing A (2017) A Cut Discontinuous Galerkin Method for Coupled BulkSurface Problems. ArXiv e-print ArXiv:1707.02153v1 [math.NA]

Pichot G, Erhel J, de Dreuzy JR (2012) A generalized mixed hybrid mortar method for solving flow in stochastic discrete fracture networks. SIAM J Sci Comput 34(1):B86-B105

Reichenberger V, Jakobs H, Bastian P, Helmig R (2006) A mixed-dimensional finite volume method for two-phase flow in fractured porous media. Adv Water Resour 29(7):1020-1036

Sandve TH, Berre I, Nordbotten JM (2012) An efficient multi-point flux approximation method for Discrete Fracture-Matrix simulations. J Comput Phys 231(9):3784-3800

Schwenck N, Flemisch B, Helmig R, Wohlmuth BI (2015) Dimensionally reduced flow models in fractured porous media: crossings and boundaries. Comput Geosci 19(6):1219-1230 